\title{
Genome-wide replication landscape of Candida glabrata
}

\author{
Stéphane Descorps-Declère ${ }^{4 \dagger}$, Cyril Saguez ${ }^{1,2,3 \dagger}$, Axel Cournac ${ }^{2,5}$, Martial Marbouty ${ }^{2,5}$, Thomas Rolland ${ }^{6}$, \\ Laurence $\mathrm{Ma}^{7}$, Christiane Bouchier ${ }^{7}$, Ivan Moszer ${ }^{8}$, Bernard Dujon ${ }^{1,2,3}$, Romain Koszul ${ }^{2,5}$ and Guy-Franck Richard ${ }^{1,2,3^{*}}$
}

\begin{abstract}
Background: The opportunistic pathogen Candida glabrata is a member of the Saccharomycetaceae yeasts. Like its close relative Saccharomyces cerevisiae, it underwent a whole-genome duplication followed by an extensive loss of genes. Its genome contains a large number of very long tandem repeats, called megasatellites. In order to determine the whole replication program of the C. glabrata genome and its general chromosomal organization, we used deep-sequencing and chromosome conformation capture experiments.

Results: We identified 253 replication fork origins, genome wide. Centromeres, HML and HMR loci, and most histone genes are replicated early, whereas natural chromosomal breakpoints are located in late-replicating regions. In addition, 275 autonomously replicating sequences (ARS) were identified during ARS-capture experiments, and their relative fitness was determined during growth competition. Analysis of ARSs allowed us to identify a 17-bp consensus, similar to the S. cerevisiae ARS consensus sequence but slightly more constrained. Megasatellites are not in close proximity to replication origins or termini. Using chromosome conformation capture, we also show that early origins tend to cluster whereas non-subtelomeric megasatellites do not cluster in the yeast nucleus.
\end{abstract}

Conclusions: Despite a shorter cell cycle, the C. glabrata replication program shares unexpected striking similarities to S. cerevisiae, in spite of their large evolutionary distance and the presence of highly repetitive large tandem repeats in C. glabrata. No correlation could be found between the replication program and megasatellites, suggesting that their formation and propagation might not be directly caused by replication fork initiation or termination.

Keywords: 3C, ACS, ARS, Candida glabrata, Chromosome organization, Megasatellites, Replication, Yeast

\section{Background}

In the present work, replication properties of the C. glabrata genome were determined using high-throughput sequencing and chromosome conformation capture (3C). Replication origins were mapped and early firing origins were found to be clustered in the nucleus, suggesting some level of organization in the temporal replication program. When replication origins and termination sites were analyzed, no evidence for an enrichment of large tandem repeats near replication origins or termination sites was found. However, chromosomal rearrangements tended to

\footnotetext{
*Correspondence: gfrichar@pasteur.fr

${ }^{\dagger}$ Equal contributors

'Institut Pasteur, Unité de Génétique Moléculaire des Levures, Département Génomes \& Génétique, F-75015 Paris, France

${ }^{2}$ CNRS, UMR3525, F-75015 Paris, France

Full list of author information is available at the end of the article
}

be more frequent in late-replicating regions, suggesting that these regions may be more prone to breakage and rearrangement during replication.

Genome replication is the preliminary and essential step to the reproduction of any living organism. To achieve that goal, a complex dedicated machinery has been set up and propagated throughout evolution. It consists of interdependent cis- and trans-acting factors, respectively replication origins and a protein machinery involved in the catalytic process of replicating DNA. The identification of replication origins in eukaryotes started with the discovery that some autonomous DNA sequences were necessary and sufficient to replicate a plasmid in the model yeast Saccharomyces cerevisiae [1,2]. Since then, genome-wide catalogs of autonomously replicating sequences (ARS) have been established in S. cerevisiae [3, 4], Lachancea kluyveri [5], Lachancea waltii [6], Kluyveromyces lactis [7], Candida albicans [8], and Pichia pastoris 
[9]. However, not all ARSs function as bona fide chromosomal replication origins [10] and a great deal of energy has been devoted, for the last 15 years, to the systematic identification and characterization of active chromosomal origins in yeast species, using different genomic approaches. Microarray hybridization of genomic DNA probes extracted at different stages during the cell cycle identified chromosomal origins and replication termination sites in S. cerevisiae [11-13], Schizosaccharomyces pombe [14], and Lachancea kluyveri [15]. Other methods used to characterize replication origins include immunoprecipitation of origin-bound pre-replication complexes, purification of nascent-strand DNA, capture and identification of replication bubbles (reviewed in $[16,17]$ ), characterization of single-stranded DNA regions [18], or comparative genomics between closely related species [19]. More recently, an approach using deep sequencing was developed. Synchronized G1 yeast cells were released through the cell cycle, and DNA was collected at different predefined intervals and sequenced to a high enough coverage to distinguish between unreplicated DNA (sequence coverage $=1$ ) and replicated DNA (sequence coverage $=2$ ). This powerful approach was applied to three Taphrinomycotina species, Schizosaccharomyces pombe, Schizosaccharomyces octoporus, and Schizosaccharomyces japonicus [20]; four Saccharomyces sensu stricto species; and one hybrid [21]. It also confirmed origins that were previously identified in Lachancea kluyveri by microarray analysis [15]. Here, we applied a related approach to characterize chromosomal replication origins and termination sites in C. glabrata.

C. glabrata belongs to the Nakaseomyces and its genome underwent the same whole-genome duplication that occurred in S. cerevisiae ancestors [22]. It is an opportunistic pathogen involved in epithelial candidiasis and bloodstream infections in immunocompromised patients [23]. Adhesion of C. glabrata to human epithelial cells is mediated by a family of calcium-dependent adhesins, called EPA genes [24], more abundant in pathogenic species of this clade [25].

In addition to EPA genes, C. glabrata encodes 44 genes containing large tandem repeats called megasatellites [26]. These megasatellites are made of large motifs (ca. 100-400 bp long), tandemly repeated from three to 32 times, located in genes potentially involved in cellular adhesion to host cells [27]. At the present time, there is no clear explanation either for the presence of so many megasatellites in the C. glabrata genome, or for the mechanism leading to their formation and subsequent propagation, despite extensive phylogenetic analyses [28, 29].

The C. glabrata genome exhibits frequent genomic polymorphisms, including copy-number variations, chromosomal translocations, and formation of neochromosomes, some of them occurring within close proximity to megasatellites [30, 31]. Determining the complete replication program of $C$. glabrata may therefore improve our understanding of relationships between megasatellites, genomic rearrangements, and S phase replication.

\section{Results \\ Identification of chromosomal replication origins and correlations to characterized genetic features}

It was previously shown that C. glabrata does not respond to a-mating or $\alpha$-mating pheromones [32]. It was therefore impossible to use this approach to obtain a homogeneous population of G1 cells, as can be done with other yeast species. Centrifugal elutriation was therefore chosen to isolate small, unbudded, G1 daughter cells from asynchronously growing populations ("Methods"; [33]). Approximately $5 \times 10^{9}$ cells were elutriated and resuspended in fresh yeast extract peptone dextrose (YPD) medium (T0 time point). After $55 \mathrm{~min}$, the first time point (T1) was collected and following samples were collected every $5 \mathrm{~min}$ for $90 \mathrm{~min}$. Fluorescence-activated cell sorting (FACS) analysis showed that approximately $15 \%$ of elutriated cells had already partially replicated their genome because a peak corresponding to G2 DNA content was already visible at T0 (Additional file 1). This suggests that C. glabrata enters $S$ phase before starting to make any bud, and that early origins probably fire soon after mitosis exit. Single read sequences corresponding to DNA extracted from time points T0 to T6 were subsequently mapped to the CBS138 reference genome. Coverage for each nucleotide, at every time point, was plotted as compared to the coverage of the same nucleotide at T0 (Fig. 1). Several coverage peaks are already visible at $\mathrm{T} 1$, corresponding to activated origins. Coverage was monitored throughout $\mathrm{S}$ phase, until the DNA content of the whole chromosome eventually doubled. For each nucleotide of the genome, a $\mathrm{T}_{50}$ value, corresponding to the time at which this nucleotide was replicated in $50 \%$ of the cells, was calculated and plotted to identify replication origins (Fig. 2, black lines). Altogether, 253 origins were identified, spaced 31-64 kilobases $(\mathrm{kb})$ from each other (average: $49 \mathrm{~kb}$, Table 1). There was no visible correlation between GC content (Fig. 2, red lines) and replication curves (Fig. 2, black lines), origins being distributed independently of any apparent composition bias. When comparing timings of origin activation, two equal-sized populations were found, corresponding to two bursts of origins firing (Fig. 3a, Additional File 2).

Average genome-wide replication fork speeds were determined for early origins and were plotted according to their distance from origins (Fig. 3b). As expected, coverage tended to decrease with distance, because regions located far from an origin are replicated later. The average fork speed was found by linear extrapolation of fork 


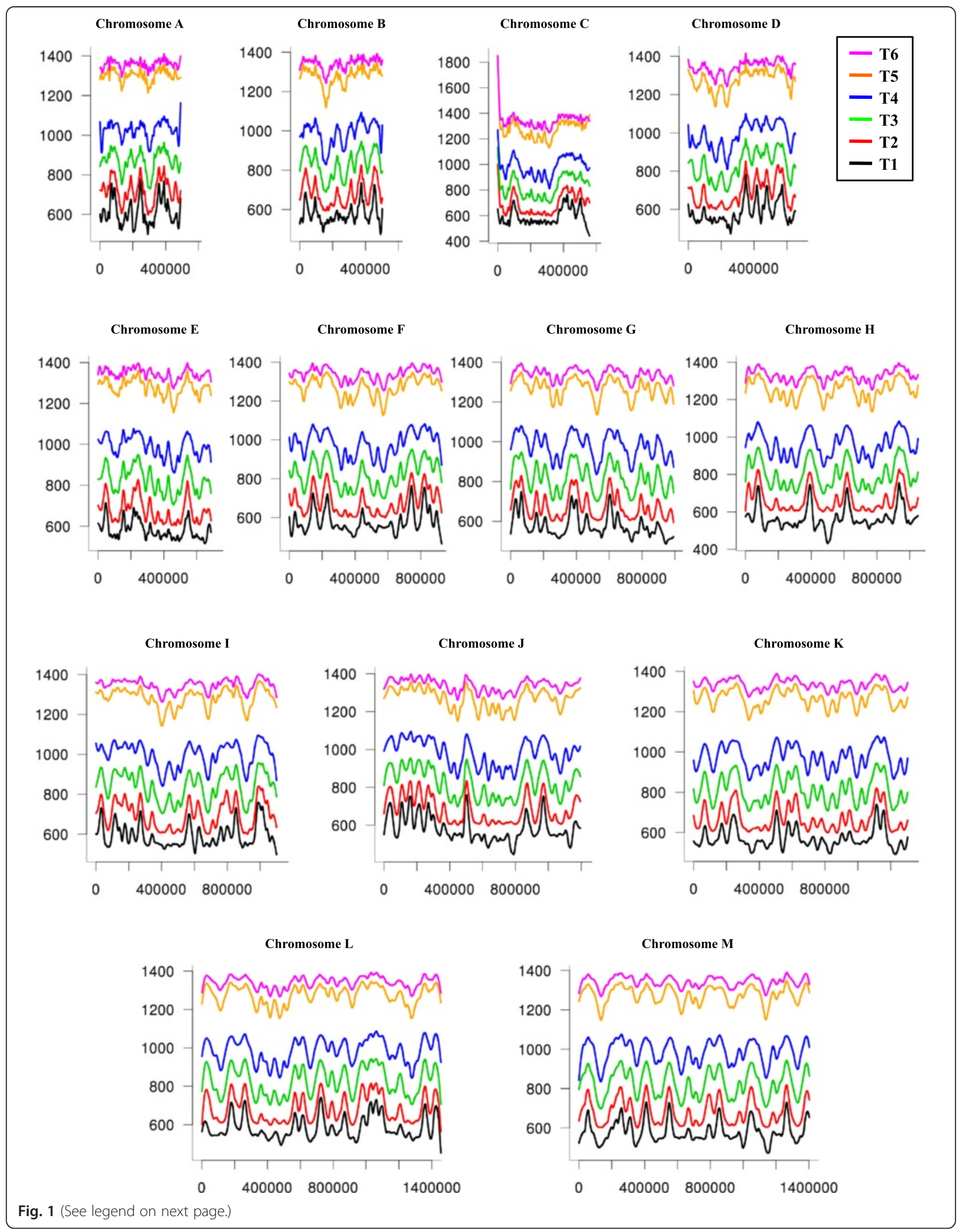


(See figure on previous page.)

Fig. 1 Sequence coverage during S-phase time course. The sequence coverage at each time point (T1 to T6) for each of the 13 chromosomes, is shown. For each nucleotide (on the $x$ axis) at every time point, relative sequence coverage is plotted (on the $y$ axis) as the ratio of Tn coverage over T0 coverage (see "Methods"). Early replication origins are visible as peaks in the first time points, and gradually disappear when regions between origins are being replicated

rates (orange or red lines) to the point at which fork rates become null (horizontal blue line). It was found to be $10.5-15.1 \mathrm{~kb}$, corresponding to an average fork speed of $2.1-3 \mathrm{~kb} / \mathrm{min}$, in the range calculated for S. cerevisiae $(2.3 \mathrm{~kb} / \mathrm{min})$ [12], L. kluyveri $(2.9 \mathrm{~kb} / \mathrm{min})$ [15], and $S$. pombe $(2.8 \mathrm{~kb} / \mathrm{min})$ [34].

All centromeres were replicated in the first burst of activation, by early origins. Telomeres were replicated in late $\mathrm{S}$ phase following the second burst of origin firing (Fig. 3c), although one telomere (chromosome $\mathrm{C}$, left arm) was replicated earlier. However, given sequencing incompleteness of C. glabrata subtelomeric regions, it is most likely that this telomere was actually replicated later on. Centromeres were always located near the very first origin firing on each chromosome, except for chromosome A (Fig. 2). Note that both donor mating-type cassettes, $H M L$ and $H M R$, were replicated at approximately the same time and before the mating-type locus MAT. Histone genes were mostly replicated early during S phase (see "Discussion").

Replication timings of known internal chromosomal deletions and chromosomal breakpoints found in translocations [30, 31] were determined. Interestingly, chromosomal breakpoints were located more often than randomly expected in late-replicating regions (seven occurrences out of

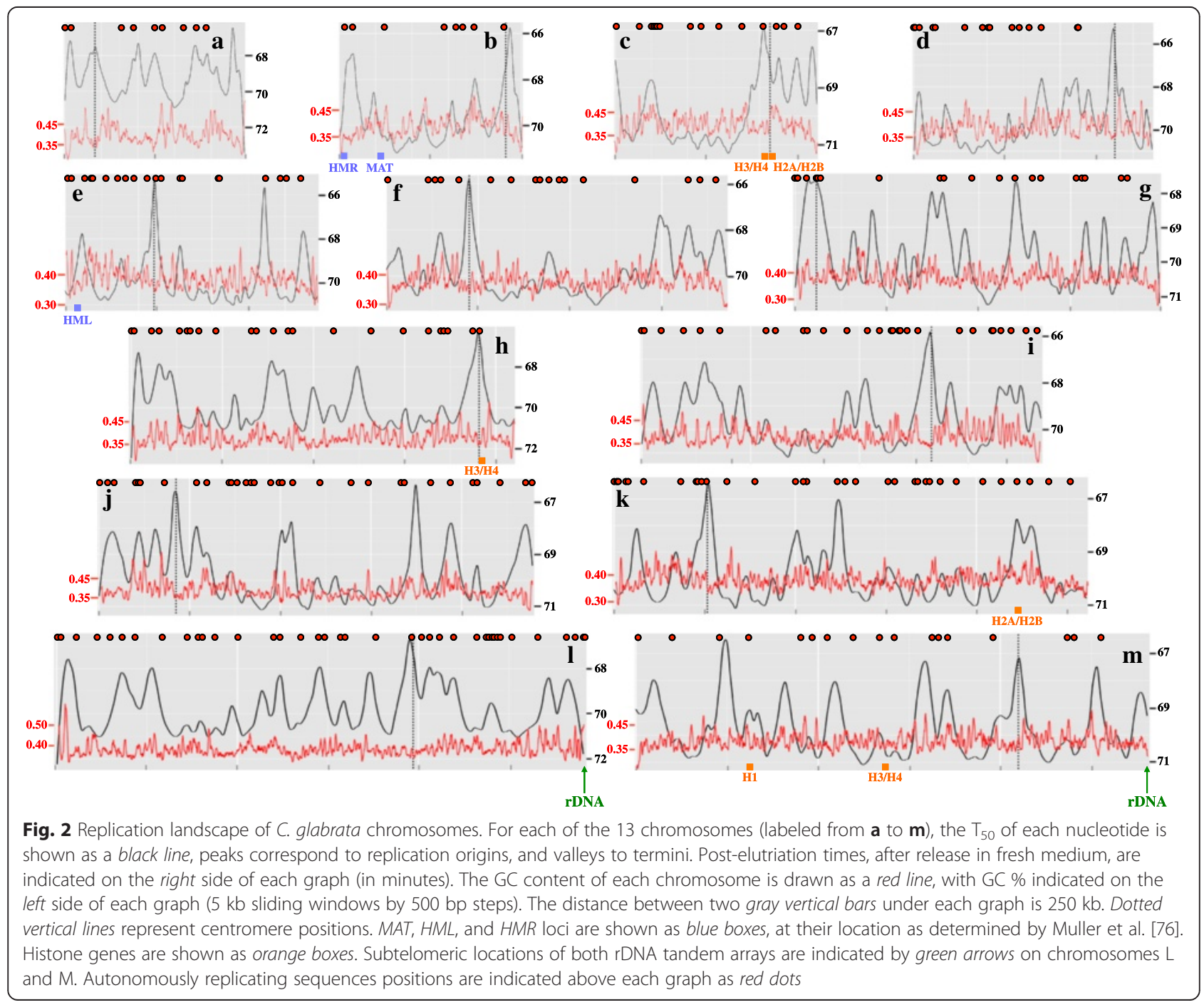


Table 1 General features of the C. glabrata replication

\begin{tabular}{llllll} 
landscape & \multicolumn{7}{l}{$l$} \\
\hline Chromosome & Size (kb) & Origins & kb/Ori & Terminations & $\mathrm{kb} / \mathrm{Ter}$ \\
\hline $\mathrm{A}$ & 491 & 16 & 31 & 15 & 41 \\
$\mathrm{~B}$ & 502 & 17 & 30 & 16 & 42 \\
$\mathrm{C}$ & 559 & 13 & 43 & 13 & 43 \\
$\mathrm{D}$ & 652 & 15 & 43 & 14 & 38 \\
$\mathrm{E}$ & 688 & 16 & 43 & 17 & 34 \\
$\mathrm{~F}$ & 927 & 21 & 44 & 20 & 49 \\
$\mathrm{G}$ & 992 & 19 & 52 & 18 & 52 \\
$\mathrm{H}$ & 1050 & 20 & 53 & 20 & 48 \\
I & 1100 & 20 & 55 & 19 & 58 \\
J & 1195 & 22 & 54 & 21 & 54 \\
K & 1303 & 26 & 50 & 26 & 57 \\
L & $1456^{\mathrm{a}}$ & 26 & 56 & 25 & 58 \\
M & $1403^{\mathrm{a}}$ & 22 & 64 & 22 & 56 \\
Total & 12318 & 253 & 49 & 248 & 50 \\
\hline
\end{tabular}

${ }^{a}$ Not including rDNA repeat arrays

$k b$ kilobase pairs

eight breakpoints, Fig. 3d), suggesting that they are more prone to either breakage or unfaithful repair. This was not true for internal deletions, which were as often located in early as in late-replicating regions (Fisher exact test $p$ value $=0.1364$ ).

Replication timing of megasatellites presented a bias for late regions (Fisher exact test $p$ value $=0.03146$ ), but when subtelomeric megasatellites were discarded, the remainder did not exhibit any bias regarding replication timing (Fig. 3e). One thousand independent random sets of nonsubtelomeric megasatellites were simulated. Their average distance to the closest replication origin was calculated to be $15.8 \pm 0.2 \mathrm{~kb}$, not significantly different from the observed distance to origins $(13.2 \pm 4.1 \mathrm{~kb})$ or to replication termini $(13.1 \pm 4.4 \mathrm{~kb})$. Therefore, megasatellites do not appear to be linked to the close proximity of origins or termini.

\section{Different replication timings of chromosome $\mathrm{C}$ arms}

In order to detect a possible bias toward early or late replication of a chromosome arm, similar to what has been described for L. kluyveri [15], $\mathrm{T}_{50}$ were plotted for each chromosome arm separately. No significant difference was observed between left and right arms, except for three chromosomes, B, C, and G (Fig. 4a). Chromosomes B and G were acrocentric (Fig. 2). Therefore, the observed difference in arm replication timing for these two chromosomes was probably due to the quasiabsence of origins in one arm. For chromosome C, however, the centromere was located three-quarters along the chromosome length, and its right arm replicated earlier. No difference in GC content was detected between left and right arms (Fig. 2), as compared to what was observed for L. kluyveri chromosome C left arm [15], presenting a $10 \% \mathrm{GC}$ enrichment and a much earlier replication pattern. Because hybridization between yeast species and large DNA introgressions are very common among yeasts $[35,36]$, it is possible that the two arms of C. glabrata chromosome $\mathrm{C}$ had different origins, explaining their different replication patterns. All chromosome $\mathrm{C}$ proteins were extracted and compared to Naumovozyma castellii, Kluyveromyces polysporus, and Zygosaccharomyces rouxii complete proteomes. These three species were chosen because they are the closest sequenced species outside the $C$. glabrata clade [22]. Proteome comparisons and clustering ("Methods" and Fig. 4b), showed no detectable evidence that C. glabrata chromosome $\mathrm{C}$ left and right arm proteins come from different phylogenetic origins. In support of this conclusion, mapping of ancestral centromeres by Gordon et al. [37] showed that C. glabrata CEN3 is an ancestral centromere and synteny is conserved across it, a result hardly compatible with chromosomal fusion of two different chromosomes. It must be concluded that the difference in replication timing observed for these two chromosomal arms is therefore not due to different phylogenetic origins.

\section{Whole-genome identification of ARSs}

ARSs were originally defined as DNA sequences providing extrachromosomal maintenance of plasmids in $S$. cerevisiae $[2,10]$. Since then, ARSs have been characterized in many yeast species [3-9]. In order to identify C. glabrata ARSs, a complete genomic library of small DNA fragments (ca. $200 \mathrm{bp}$ ) was cloned in a plasmid containing the C. glabrata URA3 gene but lacking a replication origin ("Methods"). In a first experiment, the library was transformed in a strain in which URA3 was entirely deleted (strain HM100 [32]) and 1,558 transformant colonies were obtained. Plasmidic DNA was extracted from these colonies and deep-sequenced. Using this approach, 180 different DNA fragments were mapped to the C. glabrata genome, ranging in size from 35 to 493 nucleotides (nt) (mean $=240 \pm 14 \mathrm{nt})$. In a second independent experiment, 2,616 transformant colonies were obtained and 227 fragments were mapped, ranging from 36 to $480 \mathrm{nt}$ (mean = $215 \pm 13 \mathrm{nt}$ ), 132 fragments being common to both experiments. Altogether, 275 DNA fragments able to propagate a plasmid in C. glabrata were identified. Given that 132 fragments were common to both sets of ARSs, we estimated that the C. glabrata genome should contain a total of 310-320 ARSs. During the second experiment, the library was also transformed in S. cerevisiae cells and 274 fragments were mapped (mean size $=205 \pm 9 \mathrm{nt})$. 


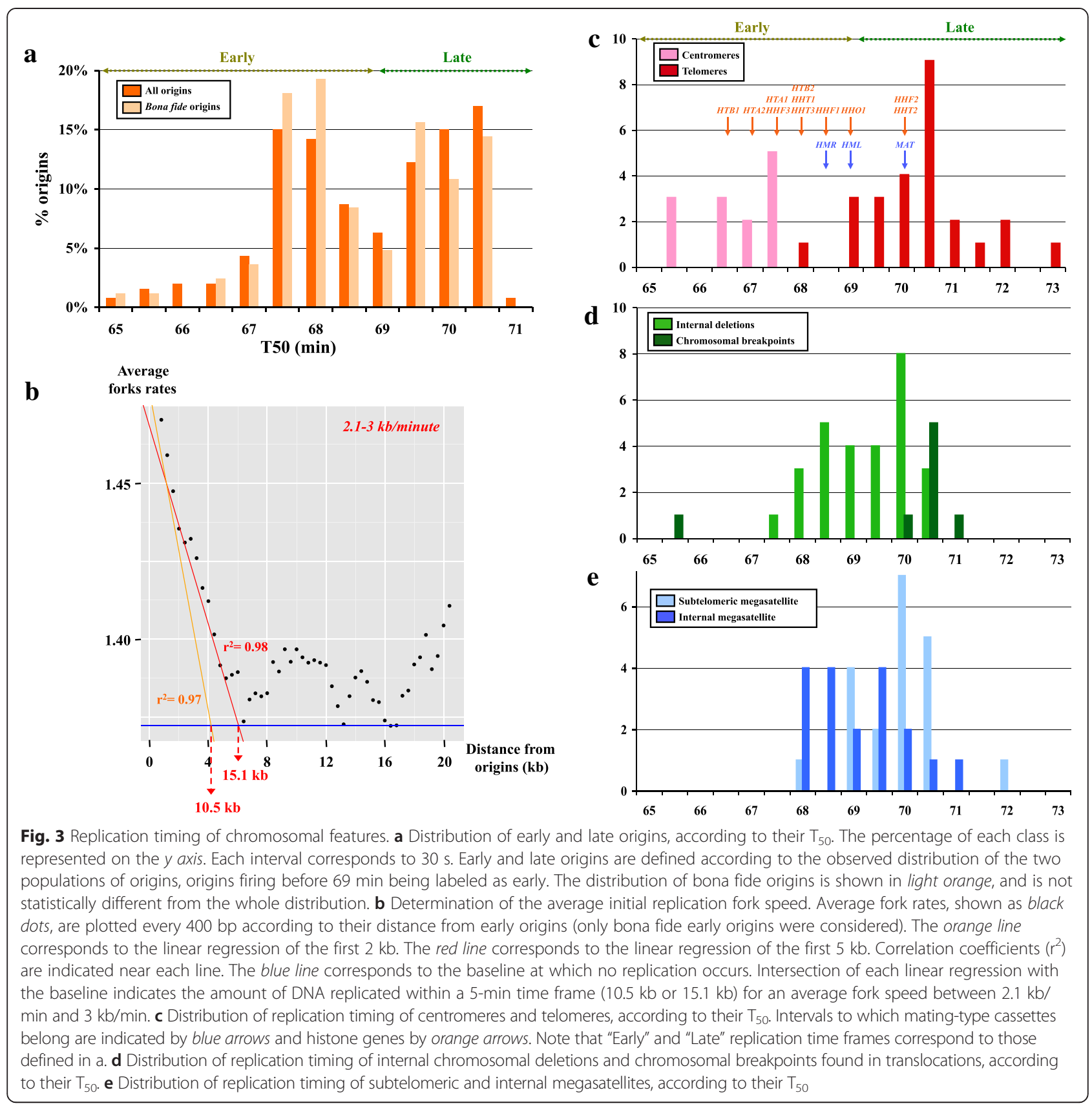

ARSs were numbered on each chromosome, according to their position, from the left to the right telomere. ARS positions were compared to replication origins, and the distance between each ARS and the closest replication origin was computed. About half of ARSs (150/275, $55 \%$ ) were found within $10 \mathrm{~kb}$ of a replication origin (Fig. 5a, blue bars ), with a majority of those within $3 \mathrm{~kb}$ (83/150). The set of origins close to these 83 ARSs were considered as bona fide origins and were used for subsequent statistical analyses. As a control, 1,000 random sets of 275 DNA fragments (random ARSs) were generated genome wide. The same number of random ARSs as compared to real ARSs was generated per chromosome, and their distance to the closest origin was calculated. The average result of these 1,000 simulations followed a normal distribution (mean $=18.5 \mathrm{~kb}$, sigma $=3.3 \mathrm{~kb}$ ) and was strikingly different from the observed distribution (Fig. 5a, gray bars).

In order to estimate replication peak resolution, we amplified, cloned in a URA3-plasmid, and transformed in C. glabrata, eight regions of $4 \mathrm{~kb}$ surrounding origins in which no ARS was detected. As a positive control, a 


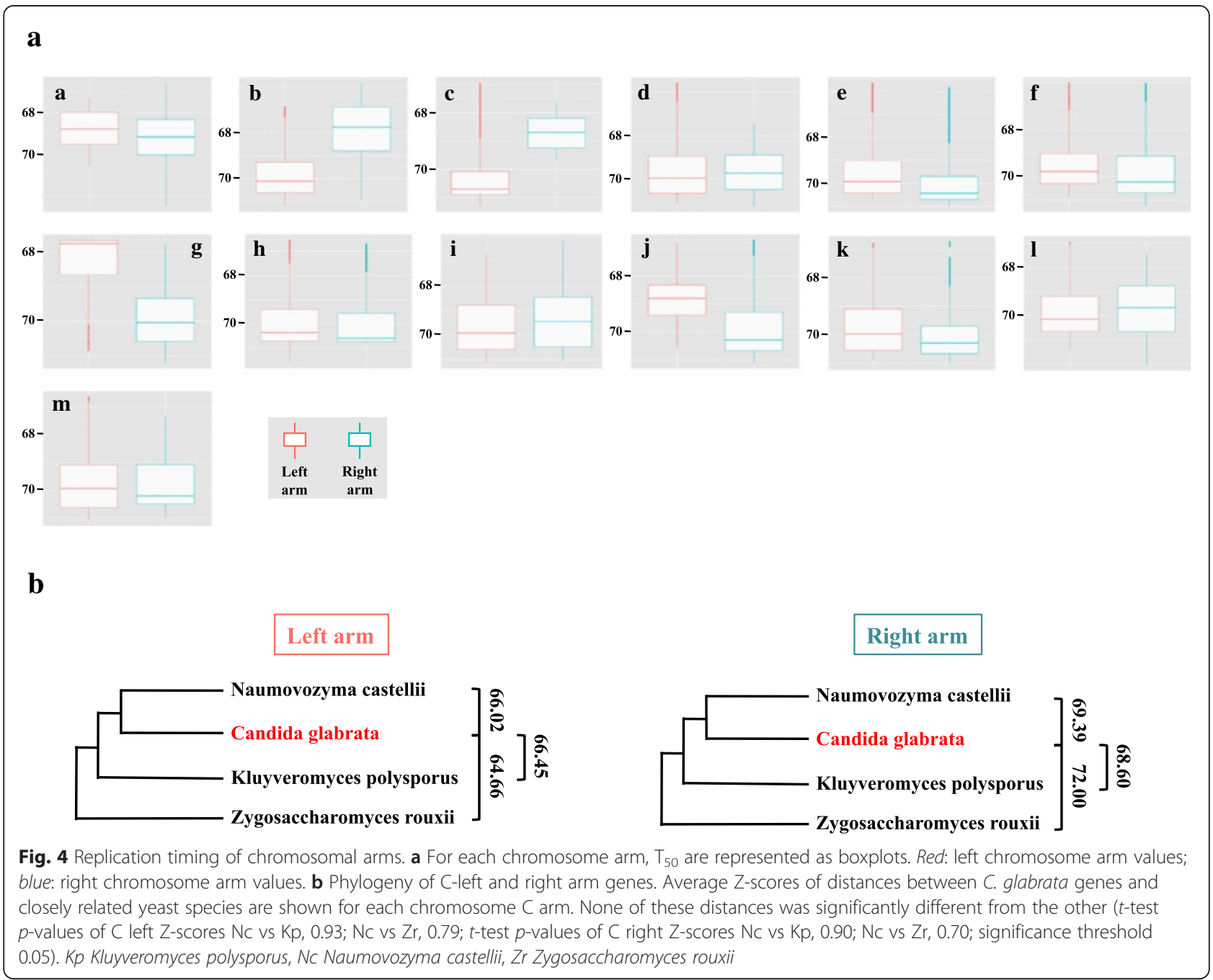

bona fide origin on chromosome $G$ containing an ARS (ARS_G11) was also cloned and transformed. Growth of the transformed cells was estimated after 1 and 4 days, in liquid culture, at $30{ }^{\circ} \mathrm{C}$, in synthetic complete (SCUra) synthetic medium. The positive control as well as two plasmids showed normal growth in these conditions. Two plasmids showed slow growth and would not have been detected in the ARS capture experiment, and four plasmids did not grow at all (Additional file 3). Therefore, only two out of eight 4-kb regions surrounding a replication peak were able to propagate a plasmid, showing that peak resolution is above $2 \mathrm{~kb}$, a conclusion already suggested by the average distance found between peaks and ARSs (Fig. 5a). This was confirmed by a second independent replication peak determination, in which average distance between bona fide origins found in both experiments was $6.9 \pm 2.4 \mathrm{~kb}$ (Additional file 4).

Using the motif finder GIMSAN, we identified a 17-bp A/T-rich ARS consensus sequence (ACS) common to the 275 DNA fragments selected (Fig. 6a). This ACS is similar to the core S. cerevisiae 11-bp ACS [10] and the 17-bp extended ACS [38], except for slight differences on three positions. When only the set of 83 bona fide origins was considered, a nearly identical motif was detected, with boundaries clearly visible (Fig. 6a). The same analysis was performed with ARSs extracted from $S$. cerevisiae. The motif detected was closer to the C. glabrata ACS than to the canonical S. cerevisiae ACS (Fig. 5b). However, when the B1 sequence was considered, particularly the WTW trinucleotide [38], it was found to be more conserved in ARSs replicating in $S$. cerevisiae than in ARSs replicating in C. glabrata. Altogether these observations strongly suggest that the requirement for the B1 box is weaker in C. glabrata, and that the information necessary to initiate replication is mostly contained in the $17 \mathrm{bp}$ ACS, in which some positions are more constrained than in the S. cerevisiae ACS. 


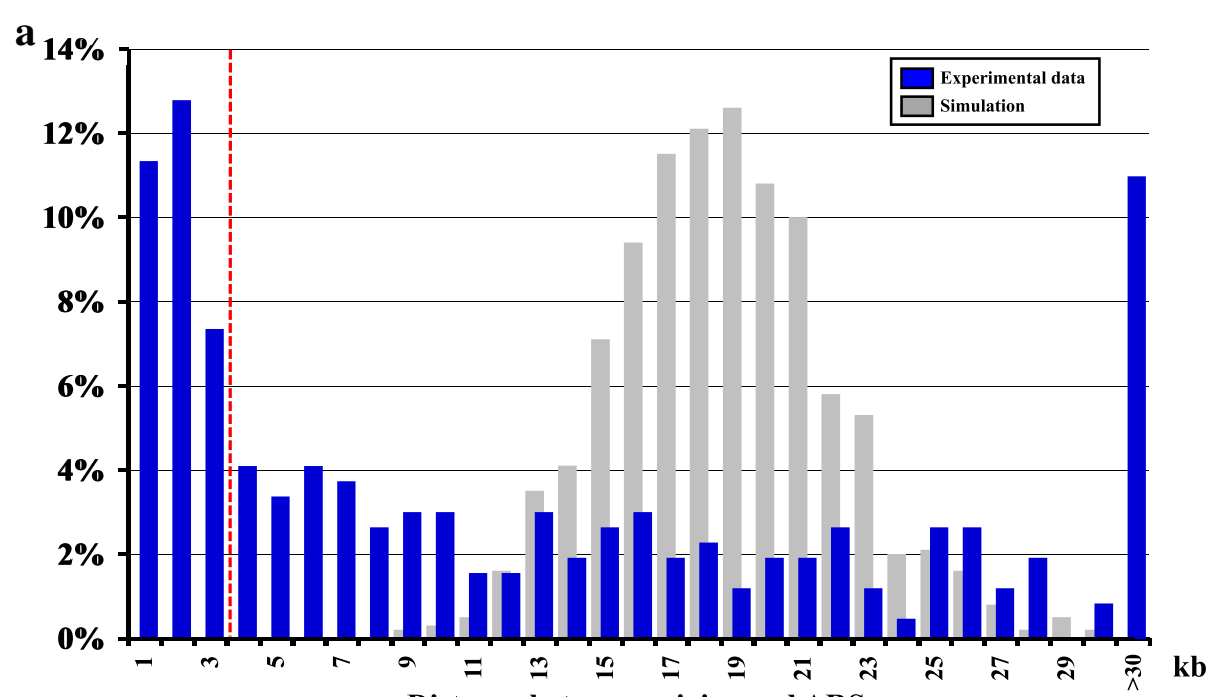

b

Distance between origins and ARSs
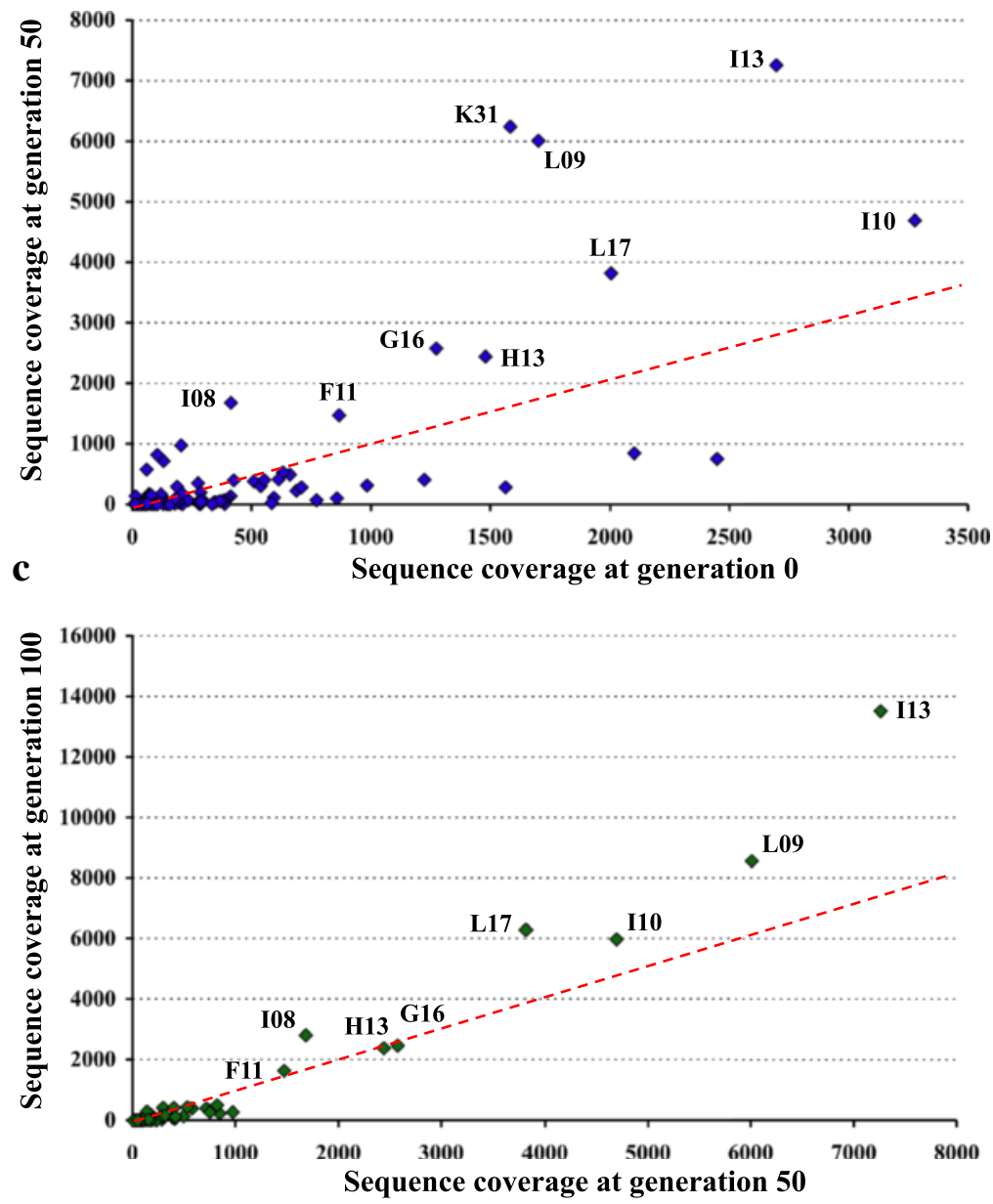

Fig. 5 ARS capture and fitnesses. a Distribution of distances between ARSs and replication origins. The observed distribution is shown in blue. The simulation of 1,000 independent experiments is shown in gray (see text). The dotted red line corresponds to the 3-kb distance limit that was chosen to define bona fide origins. b Coverage of each ARS at G50 (y axis) as compared to G0 (x axis). The dotted red line corresponds to a coverage ratio 1/1. ARSs with the highest G50 coverage are labeled. c Same as b, but for G100 coverage of each ARS (y axis), as compared to G50 coverage ( $x$ axis). ARS autonomously replicating sequence 


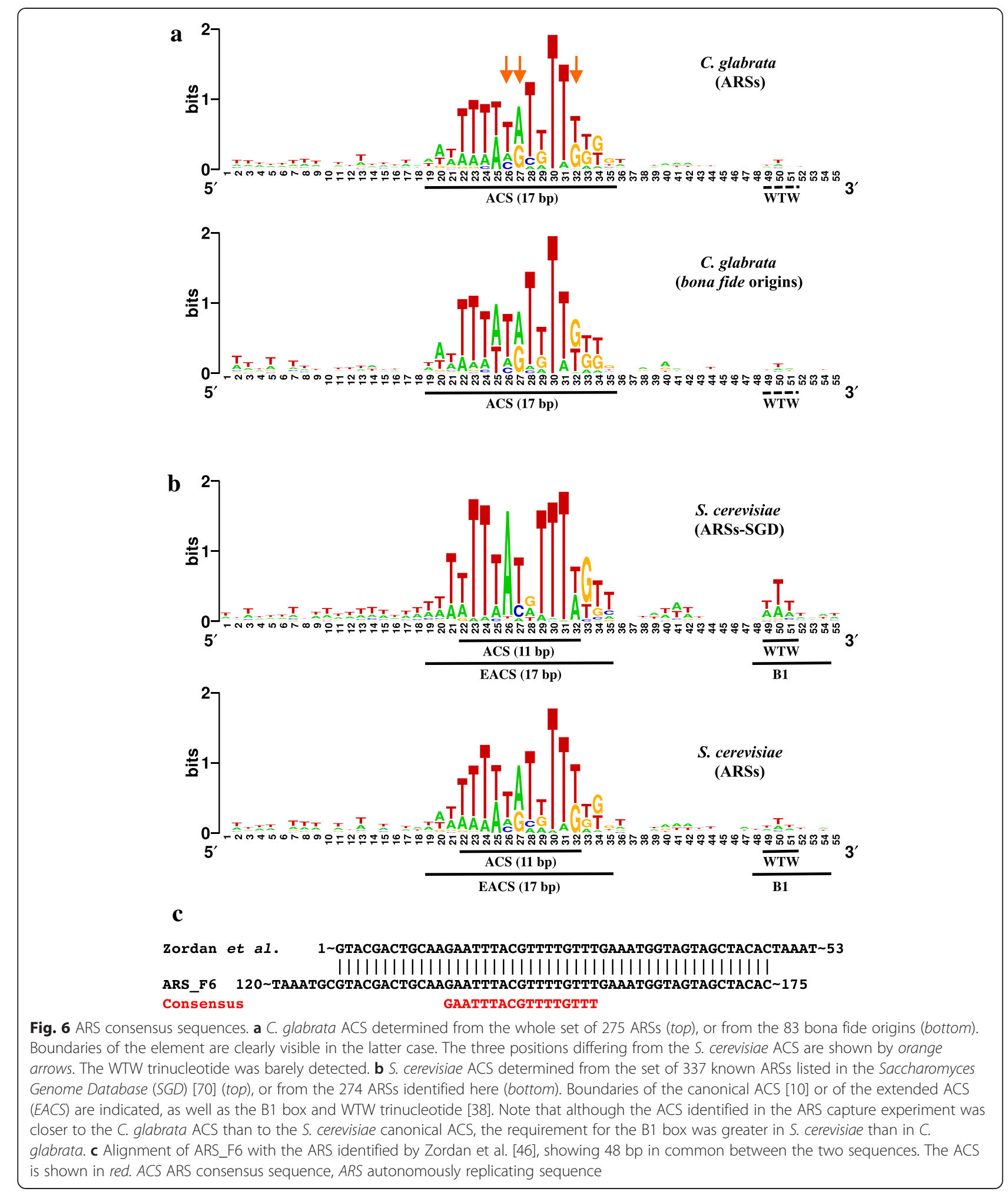

\section{rDNA replication}

In $S$. cerevisiae, rDNA tandem repeat units are carried by chromosome XII, which contains 100-200 tandem units per genome $[39,40]$. One ARS is present in each
rDNA repeat unit (ARS1200), and is located between the $18 \mathrm{~S}$ and the 5S rDNA genes (Additional file 5). In $C$. glabrata, rDNA tandem repeat units are found on two different chromosomes, $\mathrm{L}$ and $\mathrm{M}$, both in subtelomeric 
positions [41]. Two ARSs were detected in S. cerevisiae, ARS_L38 and ARS_L39, both falling in the same area as ARS1200, between the 18 S and 5S genes (Additional file 5). Coverage of the ARS library before transformation was determined to be $776 \pm 11$ for ARS_L38. Given that the library mean coverage was $9 \times$ (Additional file 6), we concluded that this region was represented in $86 \pm 1$ copies in the genome. This figures the average number of rDNA tandem repeat units in the C. glabrata genome, corresponding to 40-50 repeat units per chromosome. This number was slightly lower than in S. cerevisiae, but budding yeast contains an exceptionally high number of rDNA copies as compared to other fungi [39].

\section{ARS fitness}

In order to determine replication efficiency of each ARS, a sub-fraction (10\%) of the cell suspension, corresponding to a total 1,558 pooled colonies collected in the first ARS capture experiment, was grown for 100 additional generations in a medium selective for plasmid maintenance. DNA was extracted from cells after 50 generations (G50), or after 100 generations (G100), and deep-sequenced. Altogether, 109 independent ARSs were identified at G50, and 77 at G100 (compared to 180 ARSs at the beginning of the experiment, G0). All ARSs found at G100 were already present at G50 and all ARSs present at G50 were already present at G0 (Additional file 7). Sequencing coverage of each ARS at each generation time was considered to represent replication efficiency, because efficient ARSs should be more often represented than others. G50 coverage was plotted against G0 coverage and showed that 25 ARSs were more represented after 50 generations, nine of them exhibiting more than 1,000-fold coverage (Fig. 5b). Similarly, G100 coverage was plotted against G50 coverage, and showed that 11 ARSs were more represented after an additional 50 generations, eight out of the former nine ARSs still exhibiting more than 1,000-fold coverage. The only ARS that was no longer represented at G100 among the former nine was ARS_K31 (Fig. 5c). Mean sizes of mapped DNA fragments were $221 \pm 15 \mathrm{nt}$ and $235 \pm$ $18 \mathrm{nt}$ for G50 and G100 respectively (as compared to 195 $\pm 12 \mathrm{nt}$ for G0). This suggests that during the course of this competition experiment, plasmids that replicated more efficiently also contained a slightly larger insert size, probably to more easily accommodate the fixation of ORC and MCM complexes [42].

Absolute fitness of a given genotype was defined as the ratio of the number of individuals with that genotype at generation $\mathrm{N}+1$ to the number of individuals with the same genotype at generation $\mathrm{N}$. In replication competition experiments, efficacy of a given ARS at a given generation was considered to equal the sequence coverage of that ARS. Hence, absolute fitness of each ARS was determined as [(coverage at generation $\mathrm{N}+50) /($ coverage at generation $\mathrm{N})] / 50$. This was determined for the first 50 generations, as well as for the following 50 generations, and averaged. At G50, absolute fitness ranged from 0 (the ARS is not represented anymore after 50 generations) to 0.2196 for ARS_K06. Similarly, G100 absolute fitness ranged from 0 to 0.0433 for ARS_K06, and the average absolute fitness for G50 + G100 ranged from 0 to 0.1314 for ARS_K06. Relative fitness was calculated by giving the value 1 to the ARS with the highest average absolute fitness, other ARSs being given proportional relative fitnesses between 0 and 1 (Additional file 7).

Table 2 summarizes locations and properties of the nine ARSs exhibiting the highest coverage at G50 and G100 (as shown in Fig. 5b,c). There was no obvious correlation with distances to the closest origin, only four ARSs out of nine being located less than $2 \mathrm{~kb}$ from an origin. However, they all exhibited a relative fitness $\geq 20 \%$ (only 16 out of 180 ARSs showed a relative fitness $>20 \%$ ). Hence, any of these nine ARSs would be a good choice for an efficient multicopy plasmid replicator in C. glabrata.

\section{Chromosomal organization}

In S. cerevisiae, early replication origins exhibit enriched tridimensional contacts in the nucleus [43]. In order to

Table 2 Location and characteristics of the nine most efficient ARSs of the C. glabrata genome

\begin{tabular}{|c|c|c|c|c|c|c|c|}
\hline ARS & Start & End & Size (bp) & Closest origin & Ori-ARS (nt) & T50 & Relative fitness (\%) \\
\hline F11 & 471,720 & 472,032 & 312 & 479,174 & 7,454 & 70.49 & 21.4 \\
\hline G16 & 770,672 & 770,894 & 222 & 792,365 & 21,693 & 70.35 & 22.7 \\
\hline $\mathrm{H} 13$ & 433,274 & 433,562 & 288 & 416,726 & 16,548 & 69.67 & 20.0 \\
\hline 108 & 343,823 & 344,156 & 333 & 342,422 & 1,401 & 69.57 & 43.8 \\
\hline 110 & 448,861 & 449,108 & 247 & 450,097 & 1,236 & 69.98 & 20.6 \\
\hline 113 & 566,761 & 567,059 & 298 & 565,468 & 1,293 & 68.37 & 34.7 \\
\hline K31 & $1,152,867$ & $1,153,097$ & 230 & $1,150,055$ & 2,812 & 68.02 & 30.0 \\
\hline L09 & 359,511 & 359,818 & 307 & 360,585 & 1,074 & 70.50 & 37.8 \\
\hline L16 & 725,573 & 725,932 & 359 & 666,139 & 6,176 & 70.44 & 27.1 \\
\hline
\end{tabular}

ARS autonomously replicating sequence, $b p$ base pairs, $n t$ nucleotides 
characterize whether this organization could be reproduced in another ascomycete, a genomic $3 \mathrm{C}$ experiment was performed on C. glabrata. As previously described for $S$. cerevisiae, the C. glabrata contact map exhibits a typical Rabl pattern, with centromeres as well as telomeres presenting highly significant clustering [44] (Fig. 7a). A contact score, reflecting the enrichment in contacts between regions of interest as compared to a null model, was computed for early and late replication origins (Fig. 7b and Additional file 8) [45]. Early replication origins presented significantly enriched contacts, in contrast to late origins, similar to what was observed in S. cerevisiae [43]. The association of megasatellites with subtelomeric regions resulted in a clustering effect, but megasatellites in non-subtelomeric regions did not exhibit any enrichment in contacts (data not shown).

\section{Discussion}

In the present work, we identified 253 replication origins and 275 ARSs in the pathogenic yeast C. glabrata. These numbers are comparable to those found in other Saccharomycotina yeasts like S. cerevisiae (247-332 origins $[12,13]$ and 337 ARSs), L. kluyveri (220 origins [15] and 84 ARSs), and L. waltii (195 origins and 183 ARSs [6], or Taphrinomycotina yeasts like S. pombe (143-387 origins [20], and S. octosporus (208 origins [20]), but are significantly lower than in S. japonicus (526-542 origins [20]. In other yeast species studied, most replication origins fall within intergenic regions. Here, all the 83 bona fide origins were located in intergenes, confirming the strong bias observed in other yeast species $[6,7,9]$.

Only one ARS was previously identified in the $C$. glabrata genome, on chromosome F (286,060-286,210), in strain BG2, and used to build a series of replicating $C$. glabrata plasmids [46]. A replication peak was found on chromosome $\mathrm{F}$ at position 286,761, and an ARS was detected at 285,933-286,107 (ARS_F6). When both ARS sequences were aligned, $48 \mathrm{bp}$ were found in common between the two sequences. Right in the middle of these $48 \mathrm{bp}$ an ACS was detected (Fig. 6c). The 17-bp A/Trich ACS detected here is very similar to the ACS described for S. cerevisiae [10, 38], showing that despite the evolutionary distance separating these two yeast species [22], binding specificities of ORC/MCM complexes are probably conserved. This is consistent with a former study showing the high level of conservation of proteins involved in replication between S. cerevisiae and C. glabrata [47]. Similarly, an A/T-rich consensus was found in L. kluyveri, L. waltii, S. paradoxus, and S. bayanus $[5,6,21]$, proving that $\mathrm{ORC/MCM}$ binding specificities are also conserved over larger evolutionary distances. Interestingly, a yeast species recently shown to be a interspecies hybrid [35] contains two different sets of ARSs. AT-rich ARSs are reminiscent of the S. cerevisiae type, whereas GC-rich
ARSs seem to be more specifically associated with early origins [9]. Analysis of 69 ARSs cloned from Kluyveromyces lactis showed that the ACS was longer (50 bp) in this yeast species [7]. The authors proposed that although $K$. lactis have fewer ARSs than S. cerevisiae, the larger size of $K$. lactis ARSs allows them to encompass more features important for replication, making them more efficient than $S$. cerevisiae ARSs. It seems that $C$. glabrata, with its large number of ARSs, is closer to the S. cerevisiae paradigm than to K. lactis.

\section{Fragile sites and replication origins}

Fragile sites on metaphasic chromosomes in human cells are defined as regions of chromatin constriction or double-strand broken chromatid(s) that occur when cells are grown in the presence of drugs impeding replication (reviewed in $[48,49]$ ). The precise mechanism(s) for fragile site expression is not fully understood. For the common fragile site $F R A 3 B$, it was shown that fragility in lymphoblastoid cells was correlated to the lack of replication initiation in this 700-kb region, resulting in cells entering mitosis with an incompletely replicated $F R A 3 B$ locus [50]. For $F R A X A$, a rare fragile site involved in the most common cause of hereditary mental retardation, fragile X syndrome, it was recently shown that the large CGG trinucleotide repeat expansion at this locus impairs replication fork progression coming from an origin immediately adjacent to the repeat tract [51]. Hence, the current model postulates that chromosomal fragility is triggered by a paucity of nearby replication origins or by the blocking of a nearby origin by a specific DNA sequence, leading in both cases to mitosis entry with incompletely replicated chromatid(s). In S. cerevisiae, it was shown that regions containing replication fork termination sites often contain genetic elements involved in fork pausing (centromeres, tRNA genes, long terminal repeats, etc.) [11]. It was therefore suggested that these late-replicated regions would be favored sites for chromosomal breakage. In C. glabrata, eight chromosomal breakpoints that are commonly found between different strains have been precisely mapped by previous authors [30, 31]. Interestingly, chromosomal breakpoints are more often located near late-replicating origins (seven occurrences out of eight breakpoints, Fig. 3d), the only breakpoint near an early-replicating origin being a terminal truncation of chromosome F [31]. This suggests that C. glabrata chromosomes are likely to break more often in regions that may be incompletely replicated, owing to the late arrival of forks coming from late-firing origins. That observation will be interesting to confirm when more rearrangement breakpoints are precisely characterized to obtain significant numbers. In $L$. kluyveri and $L$. waltii, no association between synteny breakpoints and replication origins could be found, but 


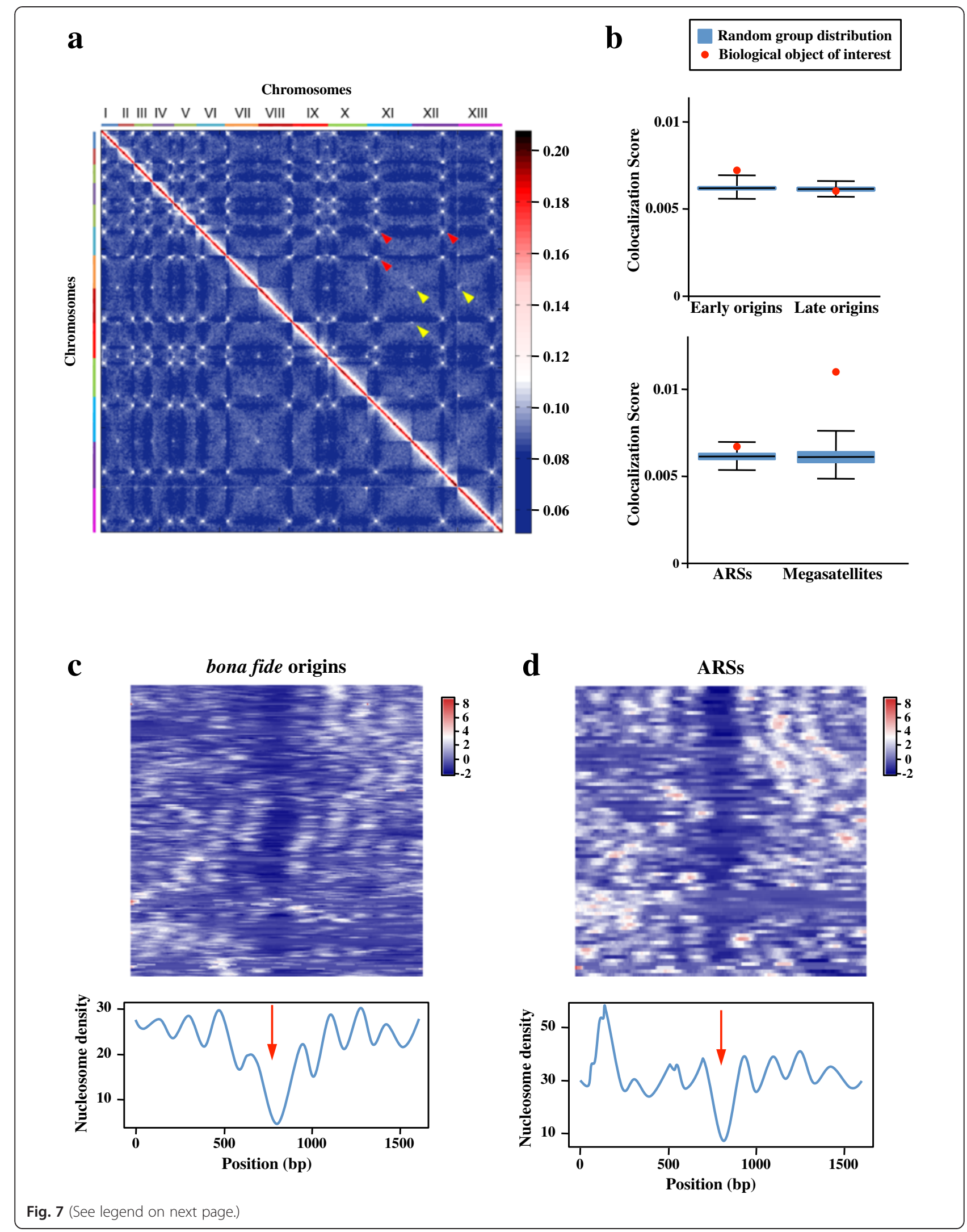


(See figure on previous page.)

Fig. 7 Chromatin organization of C. glabrata exponentially growing cells. a Normalized genomic contact matrix obtained from an asynchronous growing population. The 13 chromosomes are indicated on the $x$ axis. The color scale on the right indicates the frequency of interactions between two regions of the genome (blue rare contacts; white frequent contacts; red very frequent contacts; exponential scale). Red arrowheads centromere clustering. Yellow arrowheads telomere contacts. b Colocalization Score (CS) for DNA regions of interest. The CS is the mean of measured contacts between DNA regions. The boxplots represent the distribution of CS expected by chance, obtained with 1,000 randomized sets of positions (keeping overall chromosomal distribution), whereas the red dot corresponds to the CS of each group of interest (Megasatellites, ARSs, early and late origins). Statistical significance of colocalization is attained when the red dot lies above the random group distribution ( $p$-value $<0.001$ ). c Nucleosomal organization at bona fide origins. Nucleosome signals 800 bp upstream and downstream of bona fide origins were aligned (top), the color code representing nucleosome density (blue low density; red high density). The bottom curve represents the average value of nucleosome density. Regular nucleosome spacing is observed, with a large depletion at replication origins (red arrow). Note that ACS positions (instead of ARS/origins positions) were used, to increase resolution. $\mathbf{d}$ Nucleosomal organization at all ARSS. Nucleosome density around all ACS positions were determined and also show a significant depletion. ACS ARS consensus sequence, ARS autonomously replicating sequence

the authors did not differentiate between early and late replication origins [15], so it was impossible to compare our results.

\section{Histone genes and nucleosomes}

Highly expressed genes appear to be replicated early during $S$ phase, in yeast as well as in human cells [52]. In $S$. cerevisiae, histone-encoding genes are replicated earlier, on average, than the rest of the genome [12]. Histone expression level is also dependent on replication [53], suggesting that early duplication of histone genes leads to an early doubling of their mRNA levels, ensuring efficient DNA duplication and wrapping around histones of the rest of the genome. Indeed, replication origins near histone genes were found to be highly conserved between $S$. cerevisiae and $L$. waltii [6]. In S. cerevisiae, there are two copies of $\mathrm{H} 2 \mathrm{~A}, \mathrm{H} 2 \mathrm{~B}, \mathrm{H} 3$, and $\mathrm{H} 4$ histone genes, whereas there is only one copy of $\mathrm{H} 1$ histone gene. In C. glabrata, there are also two copies of $\mathrm{H} 2 \mathrm{~A}$ and $\mathrm{H} 2 \mathrm{~B}$ histone genes, but there are three copies each of $\mathrm{H} 3$ and $\mathrm{H} 4$ histone genes. The third copies are named CgHHT3 and CgHHF3, respectively. Our study demonstrated that histone genes are frequently located in early-replicating regions. CgHTA2, CgHTB2, CgHHT3, and $\mathrm{CgHHF} 3$ were located around the chromosome $\mathrm{C}$ centromere, and CgHHT1 and CgHHF1 were near the chromosome $\mathrm{H}$ centromere, all of them early replicating (Fig. 2 and Additional file 9). H1 histone, encoded by CgHHO1, was in an early/late region, whereas only two histone genes, $C g H H T 2$ and $C g H H F 2$, were in a latereplicating area. We conclude that histone genes are mainly located in early-replicating regions of the C. glabrata genome.

Nucleosomes are known to be depleted in yeast promoter regions. This has been shown for several yeast species, including C. glabrata [54]. Using nucleosome positioning data, we found that all 83 bona fide origins were located in nucleosome-depleted regions (Fig. 7c). The same observation was made for the 275 ARSs
(Fig. 7d). This confirms that replication origins (and ARSs) are mainly located in promoter regions.

\section{Revisiting the C. glabrata cell cycle}

Generation doubling time was determined to be 79-87 $\mathrm{min}$ (mean $83 \mathrm{~min}$ ) in our experimental conditions (SC-Ura medium, $30{ }^{\circ} \mathrm{C}$ ). Given that $\mathrm{S}$ phase itself lasts $20-25 \mathrm{~min}$ (Additional file 1 ), roughly $1 \mathrm{~h}$ is left to complete the cell cycle until the next $S$ phase. The observation that unbudded cells, freshly elutriated, already exhibited a $15 \%$ increase in DNA content suggests that the C. glabrata G1 phase of the cell cycle must be shorter than the S. cerevisiae G1 phase, with early origins probably firing shortly after mitosis exit. This may explain-at least in part-why $C$. glabrata is slightly more resistant to $\gamma$ irradiation than haploid S. cerevisiae cells (but less so than diploids) [47]. Spending less time in G1, C. glabrata would have more opportunities to repair lethal double-strand breaks using sister chromatids. This will need to be confirmed by additional dedicated experiments.

\section{Conclusions}

We determined the complete replication program of $C$. glabrata using two complementary approaches, deepsequencing of synchronized replicating cells and ARS capture. Mapping of genetic elements to replication profiles showed that natural chromosomal fragile sites are clearly located in regions of late replication, suggesting that forks tend to collapse and break more easily in these regions. No correlation could be found between the replication program and megasatellites, suggesting that their formation and propagation might not be directly caused by replication fork initiation or termination. It is striking that the ACS is conserved between C. glabrata and S. cerevisiae, despite their large evolutionary distance, suggesting that the same exact set of proteins is involved in replication initiation in both yeasts. Finally, clustering of early origins in this yeast genome raises the intriguing question of the conservation of this genomic feature among other ascomycetes. 


\section{Methods}

\section{Centrifugal elutriation}

An overnight pre-culture of CBS138 C. glabrata cells was performed in $200 \mathrm{ml} \mathrm{SC}$-Ura at $23{ }^{\circ} \mathrm{C}$. The culture was diluted to $6 \times 10^{7}$ cells $/ \mathrm{ml}$ in $800 \mathrm{ml} \mathrm{SC}$-Ura, and antifoaming agent (Antifoam 204, Sigma) was added to the culture to limit air bubble formation that could impede elutriation. Use of YPD instead of SC-Ura resulted in a high proportion of cell aggregates, making elutriation impossible. Cells were returned to growth for $2 \mathrm{~h}$ at $23{ }^{\circ} \mathrm{C}$. The large elutriation chamber $(40 \mathrm{ml}$ volume, JE-5.0 rotor, Beckman) was loaded with ca. $5 \times 10^{10}$ cells, at $23{ }^{\circ} \mathrm{C}, 3500 \mathrm{rpm}$, at a flow rate of $20-32 \mathrm{ml} / \mathrm{min}$ (increasing flow rate by $2 \mathrm{ml} / \mathrm{min}$ every $2 \mathrm{~min}$ ) in an Avanti J-26×PI elutriation centrifuge (JE-5.0 rotor, Beckman). A peristaltic pump Masterflex (L/S Digital standard drive, LC-07523-47) was used with silicone tubings (Masterflex, diameter $1 / 8^{\circ}$ in., CN-96410-16) for all subsequent loading, washing, and collecting operations. After chamber loading, equilibration was performed for $1 \mathrm{~h}$ in $1 \times$ PBS buffer, at $3500 \mathrm{rpm}$, at a flow rate of $32 \mathrm{ml} / \mathrm{min}$. After equilibration, the flow rate was increased by $2 \mathrm{ml} / \mathrm{min}$ every $2 \mathrm{~min}$, until the first cells exiting the chamber were detected, at a flow rate of 48 $\mathrm{ml} / \mathrm{min}$. From that point, cells were collected in $500 \mathrm{ml}$ fractions, checked under the microscope, and centrifuged in $50 \mathrm{ml}$ sterile polypropylene tubes. Preliminary experiments showed that, in contrast to $S$. cerevisiae, $C$. glabrata enters $\mathrm{S}$ phase before the bud may be detected on mother cells. Hence, cell collection was stopped when the first buds were detected on elutriated cells. The flow rate was increased by $2 \mathrm{ml} / \mathrm{min}$ every $500 \mathrm{ml}$ fraction, until budded cells became visible under the microscope (Olympus BH-2), at a flow rate of ca. $60 \mathrm{ml} /$ min. Altogether, ca. $8 \mathrm{l}$ of PBS-containing small unbudded yeast cells were collected and centrifuged, for a final amount of $5 \times 10^{9} \mathrm{G} 1$ cells (10\% of the initial amount of cells in culture). These cells were resuspended in $500 \mathrm{ml}$ fresh preheated YPD medium and incubated at $30^{\circ} \mathrm{C}$. At this stage it is crucial to use YPD instead of SC-Ura and to incubate at $30{ }^{\circ} \mathrm{C}$ instead of $23{ }^{\circ} \mathrm{C}$, otherwise cells re-enter cell cycle asynchronously. Collections were made at ten time points (ca. $5 \times 10^{8}$ cells for sequencing and $5 \times 10^{7}$ cells for FACS analysis), every $5 \mathrm{~min}$ after $55 \mathrm{~min}$. For FACS analysis, cells were incubated overnight in $70 \%$ ethanol, washed in $50 \mathrm{mM}$ sodium citrate $(\mathrm{pH} 7.5)$, sonicated to remove aggregates, and incubated for $1 \mathrm{~h}$ in $50 \mathrm{mM}$ sodium citrate containing $2 \mu \mathrm{M}$ Sytox Green (Invitrogen) [55]. Cells were loaded on a MACSQuant Analyser (Miltenyi Biotec) to determine relative proportions of cells in the G1 and G2 phase of the cell cycle. The relative amount of DNA at each time point was determined using the formula (G1 cells $+2 \times \mathrm{G} 2$ cells)/(G1 cells + G2 cells) [13] (Additional file 1).

\section{Library preparation for deep sequencing}

C. glabrata cells collected at six time points were digested with Zymolyase (300 $\mu \mathrm{g}, 100 \mathrm{~T}$, Seikagaku, Japan), and total genomic DNA was phenol-extracted and precipitated. Each library was made from $5 \mu \mathrm{g}$ DNA, sonicated to an average size of 500 bp (Bioruptor, maximum power (H), 30" ON/30" OFF cycles, nine cycles). DNA ends were subsequently repaired with T4 DNA polymerase (15 units, New England BioLabs, Boston, MA, USA) and Klenow DNA polymerase (5 units, New England BioLabs) and phosphorylated with T4 DNA kinase (50 units, New England BioLabs). Repaired DNA was purified on two MinElute columns (Qiagen) and eluted in $16 \mu \mathrm{l}(32 \mu \mathrm{l}$ final for each library). Addition of a 3' dATP was performed with Klenow DNA polymerase (exo-) (15 units, New England BioLabs) and home-made adapters were ligated with $2 \mu \mathrm{l}$ T4 DNA ligase (New England BioLabs, high concentration, $2 \times 10^{6}$ units $\left./ \mathrm{ml}\right)$. DNA was size-fractionated on a Pippin Prep (Sage Science) and the fraction containing 400-600 bp DNA fragments was recovered in LoBind microtubes (Eppendorf). Recovered DNA was PCRamplified with Illumina primers PE1.0 and PE2.0 and Phusion DNA polymerase (1 unit, Thermo Scientific) for nine cycles. Twenty-five PCR reactions were pooled, for each library, and purified on Qiagen purification columns (two columns were used for 25 PCR reactions). Elution was performed in $60 \mu \mathrm{l}$ (twice $30 \mu \mathrm{l}$ ) and DNA was quantified on a spectrophotometer and on an agarose gel.

\section{Analysis of Illumina reads and determination of replication origins}

Each library was run on a HiSeq 2000 (Illumina) as 50-bp single read sequences. From 112 to 146 millions reads were obtained for each library, corresponding to 466-608× coverages for the C. glabrata genome. FastX_quality_stat [56] was used to assess read quality. Because read quality was high enough (average quality $>20$ ), no read was discarded at that stage. Repeated sequences, especially megasatellites, were replaced by " $G$ " stretches in the reference sequence (C. glabrata complete genome sequence, release 10/09/ 2008, [57, 58]), before mapping was performed using $B W A$ [59]. Reads whose sequence quality was below 20 (Phred quality) were trimmed during mapping. After trimming, reads whose mapping quality was below 20 [60] were discarded (2.6-2.8\% of total reads of each time point), mpileup (SAMtools [60]) was run to determine the coverage of each nucleotide, and duplicate reads were removed by rmdup (SAMtools [60]). Coverages were finally imported in the $R$ package [61].

In order to remove under-representations and overrepresentations in each library, aberrant coverage values between (coverage median/2) and (coverage median $\times 2$ ) were filtered. Coverages were corrected for the amount of DNA measured by FACS at each time point (Additional 
file 1). Normalization was performed by multiplying each coverage value by the ratio (median coverage at time point $\mathrm{Tn} /$ median coverage at time point $\mathrm{T} 0$ ).

$\mathrm{T}_{50}$ were calculated, for each nucleotide, at each time point between $\mathrm{T} 1$ and $\mathrm{T} 6$, by plotting coverages at each time point. A four-parameter logistic non-linear regression was used to calculate the inflection point $\left(T_{50}\right)$ of each sigmoid regression:

$$
F(x)=d+\frac{a-d}{1+\left(\frac{x}{c}\right)^{b}}
$$

with $\mathrm{a}=$ minimum coverage; $\mathrm{b}=$ slope; $\mathrm{c}=$ inflection point; $\mathrm{d}=$ maximum coverage, $\mathrm{x}=$ coverage at time point Tn [62].

Finally, locally weighted scatterplot smoothing [63] was applied to smooth $T_{50}$ curves. Several span values were used, from almost no smoothing (0.0001) to extensive smoothing (0.075), and results were compared with data obtained from ARS capture experiments. This allowed us to empirically determine that a span value equal to 0.04 was the optimum (Additional file 10). Subsequently to smoothing, local maxima were computed to determine peaks corresponding to replication origins [64].

\section{Fork speeds}

Only early replication origins firing before 69 min were taken into consideration. Average ratios were calculated by normalizing sequence coverage at $\mathrm{T} 3\left(65^{\prime}\right)$ and $\mathrm{T} 4$ (70') with T0 coverage. Average rates corresponded to slopes between normalized T3 and T4 mean coverages. The same procedure was applied every $400 \mathrm{bp}$ from the origin, up to $20 \mathrm{~kb}$ away, and the corresponding 50 average rates were plotted (Fig. 3b). Linear regressions of fork rates for the first $2 \mathrm{~kb}$ (orange line) or $5 \mathrm{~kb}$ (red line) are shown. These lines were extrapolated to the baseline, at which no replication was occurring (horizontal blue line). Intersects occured at $10.5 \mathrm{~kb}$ and $15.1 \mathrm{~kb}$ distance from origins, corresponding to average fork speeds of $2.1-3 \mathrm{~kb} / \mathrm{min}$.

\section{Plasmid construction for ARS identification}

The pMEG1 plasmid was built from a pBlueScript II SK (+) backbone (Stratagène) in which the CgURA3 gene (CAGLOI03080g) was cloned. The gene was amplified from CBS138 genomic DNA, using primers CgURA3for (GGAAGATCTTCCTCCTGTAATTACAACAATTCAA) and CgURA3rev (CGGGATCCCGGTTGCCATTACGCC ACGCGAGC), amplifying a 1,597-bp fragment containing the CgURA3 gene plus 500 bp upstream and 300 bp downstream of the open reading frame. This PCR fragment was digested by BglII and BamHI and ligated to pBlueScript digested by BamHI, and dephosphorylated. The resulting pMEG1 plasmid was sequenced and no mutation was found in CgURA3 or in its flanking regions. A pMEG1-derivative pMEG1s plasmid was built by inserting phosphorylated and annealed SC126 (CCGGGAACG TAGTGACAGGTAC) and SC127 (CTGTCACTACGTT CCCGGGTAC) oligonucleotides into the unique KpnI site of pMEG1. Hence, pMEG1s carries a unique SmaI site surrounded by KpnI sites. Neither pMEG1 nor pMEG1s naturally propagate in C. glabrata.

\section{C. glabrata genomic library for ARS identification}

Genomic DNA from C. glabrata CBS138 strain was isolated as described above. We sonicated $20 \mu \mathrm{g}$ of DNA to an average size of $200 \mathrm{bp}$ on a Covaris S220 (MicroTUBE AFA Fiber; peak power 175; duty factor 10; cycles/burst 200; $280 \mathrm{~s}$ ) and fragments of size 150-250 bp were purified from a $3 \%$ MetaPhor gel (Lonza) using Qiaquick columns (Qiagen). Fragmented ends $(5 \mu \mathrm{g})$ were repaired with T4 DNA polymerase (15 units, New England BioLabs) and Klenow DNA polymerase (5 units, New England BioLabs) and phosphorylated with T4 DNA kinase (50 units, New England BioLabs). Repaired DNA fragments were purified on a Qiaquick column and eluted in $50 \mu \mathrm{l}$ Tris $\mathrm{pH}$ 8. For library construction, $160 \mathrm{ng}$ of the above purified DNA fragments were ligated into the dephosphorylated SmaI site of pMEG1s $(1.55 \mu \mathrm{g})$ with T4 DNA ligase (2,000 units, New England BioLabs). To maximize cloning efficiency, ligation products $(100 \mu \mathrm{l})$ were purified on Qiaquick columns and eluted in $50 \mu \mathrm{l}$ $10 \mathrm{mM}$ Tris $\mathrm{pH}$ 8. Ligation products were then used to transform $1 \mathrm{ml}$ of SURE2 competent Escherichia coli cells $[65,66]$. Colonies were pooled and plasmid DNA was extracted using the alkaline lysis method followed by phenol-chloroform extraction and ethanol precipitation. Approximately 450,000 clones bearing inserts were obtained, corresponding to a total library coverage of about $7 \times$ genome size.

\section{ARS identification and replication competition experiment}

To select ARS-containing plasmids, the above library was transformed into the ura3s auxotrophic C. glabrata CBS138-derivative HM100 strain (ura3 $\triangle:: K A N$ [32]) using a modified version of the lithium acetate-heat shock method [67]. Transformed cells were plated onto complete SD medium lacking uracil. After 3 days at $30^{\circ} \mathrm{C}$, 1,558 independent [URA+] clones were obtained in the first experiment and 2,616 clones were obtained in the second experiment. Clones of the first experiment were pooled (G0) and one tenth of this pool was then used for competition experiment. Briefly, cells were diluted into complete liquid SD medium lacking uracil and grown over 50 (G50) and 100 (G100) generations, by sequential dilutions every eight generations. To minimize genetic drift due to bottleneck effect during the course of selection, 
each diluted culture contained $\sim 5.7 \times 10^{8}$ cells, corresponding to an average representation of 365,000 copies of each ARS-containing plasmid.

Genomic DNAs from the initial pool (G0) and after 50 (G50) and 100 (G100) generations were prepared as described above and then used ( $5 \mu \mathrm{l}$ out of $200 \mu \mathrm{l})$ to transform $500 \mu \mathrm{l}$ of SURE2 competent $E$. coli cells. Up to $2.5 \times$ $10^{6}$ clones were obtained after transformation of each library. Colonies were pooled and plasmidic DNAs were extracted using the alkaline lysis method followed by phenol-chloroform extraction and ethanol precipitation. Inserts from each library $(\sim 3 \mu \mathrm{g})$ were PCR-amplified with SC139 (GTAAAACGACGGCCAGTGAA) and SC140 (GTAGACAAGAACCCATAGAC) primers and Phusion DNA polymerase (1 unit per reaction, Thermo Scientific) for eight cycles. PCR products were digested with KpnI (100 U, Boehringer Mannheim) and purified from agarose gel using Qiaquick columns. Libraries for deep sequencing were prepared as described above, with the exception that fractionation on Pippin Prep was omitted.

\section{ARS library sequencing}

Paired-end reads were generated on a MiSeq (Illumina). From 332,000 to $1,418,000$ reads (250 bp on each side) corresponding to $8-34 \times$ coverages, depending on the library, were obtained. Reads were treated like previously (see above). The distribution of read coverage for the initial library (before yeast transformation) is shown in Additional file 6. Contiguous positions covered at least six times, without interruption, were scored as ARSs, independently for each library. Mean coverage of each library was determined as $\Sigma$ (ARS length $\times$ coverage $) /$ genome size.

Given that the ARS library was blunt-ended cloned, it was possible that more than one insert was present in some of the replicating plasmids. To identify double inserts, paired-end reads whose reads mapped on two different chromosomes were computed. Out of the 28 double inserts identified, 25 contained one or more ACS-containing insert. As a complementary experiment, 14 plasmids corresponding to reads in which no ACS could be clearly identified by Illumina mapping were individually amplified and sequenced from both ends. All of them but one corresponded to double inserts and one to a triple insert. In each case, one of the inserts contained the ACS, confirming the previous result. Hence, in most plasmids carrying double (or triple) inserts, at least one of the inserts contains an ACS. As a final proof, seven ACS-containing inserts and eight ACS-less inserts were individually cloned in the same $U R A 3$ plasmid used for library building. All the plasmids containing an ACS were able to replicate in C. glabrata, whereas none of those containing an ACS-less fragment could propagate. The seven ARSs that efficiently propagated a plasmid were located $0.46-16.6 \mathrm{~kb}$ from the closest replication origin and are highlighted in Additional file 7.

\section{ACS consensus determination}

From ARS coordinates (at G0), sequences were extracted from the reference genome to constitute an ARS database. In order to detect conserved motifs, GIMSAN [68] was run on the ARS library. The null model used by GIMSAN was built from sequences that were not in the ARS database. Motif sizes of 8,14 , or 20 bp were used as input parameters. When the 20-bp size was used, the consensus sequence was not significant beyond $14 \mathrm{bp}$, therefore a 14-bp consensus was kept. The GIMSAN output file was reformatted in the MEME file format. The 14-bp consensus was subsequently researched in all ARS sequences extended $200 \mathrm{bp}$ upstream and downstream, using MAST [69]. Recalculation of the $S$. cerevisiae ACS was made using the same approach as for C. glabrata, using as a library the 337 ARSs identified in the budding yeast genome [70]. Subsequent comparisons of consensus between C. glabrata ARSs and bona fide origins showed that this 14-bp consensus could be slightly extended upstream and downstream to a 17-bp consensus that fit with the S. cerevisiae extended ARS consensus (Fig. 6a).

\section{Phylogenetic analyses}

All chromosome $\mathrm{C}$ proteins were extracted from the Génolevures database [71], and compared to Naumovozyma castellii, Kluyveromyces polysporus, and Zygosaccharomyces rouxii complete proteomes. Gene families containing paralogous genes were removed, so that only orthologous families containing one and only one gene in each of the four yeast species were retained for subsequent comparisons. On chromosome C left arm, 105 genes were found to be in orthologous gene families, whereas 39 orthologous gene families were found on the right arm. In each family, the distance between the C. glabrata gene and each of its three orthologues was calculated and a Z-score was determined for this distance. Average Z-scores were slightly higher for genes located on the right arm than for the left arm for all three species (Fig. 4b). However, statistical analysis of Z-score distributions did not show any significant difference between $N$. castellii and $Z$. rouxii gene distances $(t$-test, left arm $p$-value $=0.7971$, right arm $p$-value $=0.7043)$, nor between $N$. castellii and $K$. polysporus $(t$-test, left arm $p$-value $=0.9383$, right arm $p$-value $=0.9087)$.

\section{Chromosome conformation capture}

C. glabrata cells (strain CBS138) were recovered from an exponentially growing population (YPD medium). Cells were cross-linked for $10 \mathrm{~min}$ with fresh formaldehyde (3\% final concentration), pooled as aliquots of $3 \times$ 
$10^{9}$ cells, and stored at $-80{ }^{\circ} \mathrm{C}$ until use. Aliquots were thawed on ice and resuspended in $6 \mathrm{ml} 1 \times D p n I \mathrm{I}$ buffer (New England BioLabs) and 3C libraries were generated as described [72].

The 3C libraries were sheared and processed into Illumina libraries using custom-made versions of the Illumina PE adapters (Paired-End DNA sample Prep Kit, Illumina, PE-930-1001). Fragments of sizes between 400 and 800 bp were purified using a Pippin Prep apparatus (SAGE Science), PCR amplified, and paired-end sequenced on an Illumina platform (HiSeq2000; $2 \times 100 \mathrm{bp}$ ). After sequencing, PCR duplicates were collapsed using the random tag present on each of the custom-made adapters and reads were aligned using Bowtie 2 in its most sensitive mode against the C. glabrata reference genome [73]. An iterative alignment procedure similar to [74] was used. Paired reads were aligned independently. Each mapped read was then assigned to a restriction fragment. Genome-wide contact matrices were built by binning the genome into units of 30 or 60 restriction fragments, resulting in $1351 \times 1351$ and $679 \times 679$ contact maps used for the colocalization score (CS) calculation and Fig. 7, respectively. The contact maps were subsequently normalized using the sequential component normalization procedure described in Cournac et al. [45]. This procedure ensures that the sum over the column and lines of the matrix equals 1 , which reduces both noise and biases inherent to the protocol. The CS of each feature of interest corresponds to the average number on contacts found in bins which contain the feature. The statistical significance of CS was assessed using a random sampling method [43] (Cournac and Koszul, unpublished). A thousand random sets of bins were generated and the actual CS value was compared with the distribution of CS obtained under the null hypothesis.

\section{Nucleosome positioning}

The following data file from Tsankov et al. [54] was used: GSM552914_Cgla_CM_Dec0908_nucCount_100. wig. Density heatmaps of nucleosome occupancies were plotted using the $\mathrm{R}$ function heatmap3. In this function, a $\mathrm{Z}$ transformation is used to increase pattern contrast between nucleosome-depleted and nucleosome-rich areas. Hierarchical clustering was used to classify sequences.

\section{Data deposition}

Raw Illumina sequences for replication origins, as well as for ARS capture experiments were uploaded at the EBI (accession number: PRJEB8571). Scripts used for the analyses were uploaded in a GitHub repository [75].

\section{Additional files}

Additional file 1: DNA content after elutriation. A FACS analyses and determination of DNA content. The first cell collection was made 55 min after T0, because we previously determined that this was the amount of time required for $C$. glabrata cells to re-enter the cell cycle after elutriation. Subsequent collections were made at 5 min intervals. DNA was labeled with Sytox Green [56] and analyzed by FACS (MACS Quant, Miltenyi Biotec) and relative amounts of $\mathrm{G} 1$ and $\mathrm{G} 2$ cells were calculated using FlowJo. Note that at T0, there is already a detectable amount of cells that have started to replicate their genome, suggesting that replication restarts soon after mitosis, at early origins. For each time point, peaks corresponding respectively to $G 1$ and $G 2$ cells are shown, along with percentages of cells in each phase. Dotted lines represent average intensity values of $\mathrm{G} 1$ and $\mathrm{G} 2$ peaks. B Table summarizing these results. DNA content was calculated using the formula (G1 cells $+2 \times$ G2 cells)/(G1 cells + G2 cells) [13]. The curve plots DNA content according to each time point. Note that time points are spaced at $5 \mathrm{~min}$, except between T0 and T1 (55 min). Genomic DNA from time points shaded in gray were extracted and sequenced. C FACS profiles of the first elutriated fraction of two independent experiments (TO). The small peak corresponds to cells that have already started DNA replication, although no bud is visible by visual examination. (PDF $230 \mathrm{~kb}$ )

Additional file 2: Position and firing time of each replication origin, by chromosome. Coordinates are given according to Genolevures sequence release 10 September 2008 (www.genolevures.org). Bona fide origins are in red (see text). (DOC $154 \mathrm{~kb}$ )

Additional file 3: Plasmid replication efficacy of DNA regions surrounding identified replication origins. (DOC $40 \mathrm{~kb}$ )

Additional file 4: Additional experiment performed to confirm replication origins. The time course shown in this experiment was performed as described in "Methods" for the first experiment (Additional file 1). Top: FACS analyses and DNA content are shown. Time points T0 to T6 were sequenced on a MiSeq (Illumina) sequencer and 2.3-4.1 millions reads were obtained (175 bp single reads) for each time point. Bottom: Sequence coverage for time points T2, T3, T4, and T6, for each chromosome (smoothing span: 0.05-0.09, depending on chromosome size). Sequence mapping of two time points (T1 and T5) exhibited too many gaps to allow us to calculate non-linear regressions for each of the 12 millions nucleotides of the C. glabrata genome, in order to determine T50 values. However, using T6 and T0 coverage, which correspond respectively to $S$ and $\mathrm{G} 1$ phases of the cell cycle, we were able to determine replication peak positions. A comparison of peaks detected in experiment \#1 (Fig. 2) and experiment \#2 (the present figure) was made in the bottom right table. Out of 83 bona fide origins, 73 were found in experiment \#2 (88\%), and 241 replication origins were detected (instead of 253 in experiment \#1). The average distance between bona fide replication peaks found in both experiments was $6.9 \pm 2.4 \mathrm{~kb}$ (95\% confidence interval), consistent with what was deduced from comparisons between ARS positions and replication peaks (Fig. 5a). (PDF 1224 kb)

Additional file 5: rDNA locus replication. Top: One tandem repeat unit of the S. cerevisiae rDNA locus on chromosome 12 is represented. Coordinates are shown in boxes, according to the Saccharomyces Genome Database (release 19 November 2012). The ARS present in each repeat unit is indicated as a blue diamond. Bottom: Same representation for the C. glabrata rDNA locus. Coordinates are shown according to Génolevures database (release 10 September 2008). The two ARSs captured are shown by blue diamonds. (PDF $65 \mathrm{~kb}$ )

Additional file 6: ARS library. Number of ARSs ( $y$ axis) for each coverage ( $x$ axis) in the ARS library, before transformation. Coverage was determined by Illumina sequencing (see "Methods"). The average coverage of the library was $9 \times$. Each bar represents $2 \times$ coverage. (PDF 98 kb)

Additional file 7: Positions, coverages, and fitnesses of ARSs. (XLS $69 \mathrm{~kb}$ ) Additional file 8: Colocalization scores calculated from a second $C$. glabrata $3 \mathrm{C}$ matrix [77] for each genetic element studied. Using this data set, in addition to early origins, a set of 275 ARSs colocalize. See Fig. 7 for legend. Note that in this experiment, cells were cross-linked for $30 \mathrm{~min}$ (instead of $10 \mathrm{~min}$ ) with fresh formaldehyde (3\% final concentration). (PDF $35 \mathrm{~kb}$ ) 
Additional file 9: Replication timing of histone genes. (DOC $49 \mathrm{~kb}$ ) Additional file 10: Effect of smoothing on replication curve shape. The chromosome $\mathrm{G}$ replication curve is represented after smoothing with different span values, from 0.0001 (almost no smoothing) to 0.075 (extensive smoothing). With low levels of smoothing, several peaks or shoulders did not correspond to ARS positions and were clearly artifacts (0.015 span value). This effect was particularly pronounced for small chromosomes. With extensive smoothing (0.075 span value), close replication peaks tended to merge into one single peak, like, for example, the large left-most double (or triple) replication peak. Comparison of different smoothing levels with ARS positions (red dots) led us to use a 0.04 span value for all chromosomes. (PDF $1101 \mathrm{~kb}$ )

\section{Abbreviations}

3C: chromosome conformation capture; bp: base pair; ACS: ARS consensus sequence; ARS: autonomously replicating sequence; CS: colocalization score; FACS: fluorescence-activated cell sorting; kb: kilobase pair; nt: nucleotide; SC-Ura: synthetic complete Ura medium; YPD: yeast extract peptone dextrose.

\section{Competing interests}

The authors declare that they have no competing interests.

\section{Authors' contributions}

GFR and CS conceived of and designed the replication experiments. RK and MM designed the 3 C experiments. CS, MM, LM, and GFR performed experiments. SDD and AC analyzed and interpreted sequencing and $3 C$ results, and carried out all informatics and statistical analyses. TR participated in the early analysis of sequencing data. IM, CB, and BD provided essential support and reagents to perform and analyze experiments. CS, RK, TR, and GFR wrote the manuscript. All authors read and approved the final manuscript.

\section{Author details}

'Institut Pasteur, Unité de Génétique Moléculaire des Levures, Département Génomes \& Génétique, F-75015 Paris, France. ${ }^{2}$ CNRS, UMR3525, F-75015 Paris, France. ${ }^{3}$ Sorbonne Universités, UPMC Univ Paris 06, 4 Place Jussieu, 75252, Paris Cedex 05, France. ${ }^{4}$ Institut Pasteur, Center of Bioinformatics, Biostatistics and Integrative Biology (C3BI), F-75015 Paris, France. ${ }^{5}$ Institut Pasteur, Groupe Régulation Spatiale des Génomes, Département Génomes \& Génétique, F-75015 Paris, France. ${ }^{6}$ Present address: Institut Pasteur, Unité de Génétique Humaine et Fonctions Cognitives, Département des Neurosciences, F-75015 Paris, France. ${ }^{7}$ Institut Pasteur, Plate-forme Génomique, Département Génomes \& Génétique, F-75015 Paris, France. ${ }^{8}$ Present address: Plate-forme Bio-informatique/Biostatistique, Institut de Neurosciences Translationnelles IHU-A-ICM, Hôpital Pitié-Salpêtrière, 47-83 bd de l'Hôpital, 75561, Paris Cedex 13, France.

Received: 31 March 2015 Accepted: 5 August 2015

Published online: 02 September 2015

\section{References}

1. Chan CS, Tye BK. Autonomously replicating sequences in Saccharomyces cerevisiae. Proc Natl Acad Sci U S A. 1980;77:6329-33.

2. Brewer BJ, Fangman WL. The localization of replication origins on ARS plasmids in S. cerevisiae. Cell. 1987;51:463-71.

3. Poloumienko A, Dershowitz A, De J, Newlon CS. Completion of replication map of Saccharomyces cerevisiae chromosome III. Mol Biol Cell. 2001;12:3317-27.

4. Donato JJ, Chung SC, Tye BK. Genome-wide hierarchy of replication origin usage in Saccharomyces cerevisiae. PLoS Genet. 2006;2, e141.

5. Liachko I, Tanaka E, Cox K, Chung SC, Yang L, Seher A, et al. Novel features of ARS selection in budding yeast Lachancea kluyveri. BMC Genomics. 2011;12:633

6. Di Rienzi SC, Lindstrom KC, Mann T, Noble WS, Raghuraman MK, Brewer BJ. Maintaining replication origins in the face of genomic change. Genome Res. 2012;22:1940-52.

7. Liachko I, Bhaskar A, Lee C, Chung SC, Tye BK, Keich U. A comprehensive genome-wide map of autonomously replicating sequences in a naive genome. PLoS Genet. 2010;6, e1000946.
8. Tsai HJ, Baller JA, Liachko I, Koren A, Burrack LS, Hickman MA, et al. Origin replication complex binding, nucleosome depletion patterns, and a primary sequence motif can predict origins of replication in a genome with epigenetic centromeres. MBio. 2014;5:e01703-01714.

9. Liachko I, Youngblood RA, Tsui K, Bubb KL, Queitsch C, Raghuraman MK, et al. GC-rich DNA elements enable replication origin activity in the methylotrophic yeast Pichia pastoris. PLoS Genet. 2014;10, e1004169.

10. Newlon CS, Theis JF. The structure and function of yeast ARS elements. Curr Opin Genet Dev. 1993;3:752-8.

11. Fachinetti D, Bermejo R, Cocito A, Minardi S, Katou Y, Kanoh Y, et al. Replication termination at eukaryotic chromosomes is mediated by Top2 and occurs at genomic loci containing pausing elements. Mol Cell. 2010;39:595-605.

12. Raghuraman MK, Winzeler EA, Collingwood D, Hunt S, Wodicka L, Conway A, et al. Replication dynamics of the yeast genome. Science. 2001;294:115-21.

13. Yabuki N, Terashima H, Kitada K. Mapping of early firing origins on a replication profile of budding yeast. Genes Cells. 2002;7:781-9.

14. Heichinger C, Penkett CJ, Bähler J, Nurse P. Genome-wide characterization of fission yeast DNA replication origins. EMBO J. 2006;25:5171-9.

15. Agier N, Romano OM, Touzain F, Cosentino Lagomarsino M, Fischer G. The spatiotemporal program of replication in the genome of Lachancea kluyveri. Genome Biol Evol. 2013;5:370-88.

16. Gilbert DM. Evaluating genome-scale approaches to eukaryotic DNA replication. Nat Rev Genet. 2010;11:673-84.

17. Vengrova S, Dalgaard JZ. DNA Replication: Methods and Protocols. New York: Humana Press; 2009.

18. Feng W, Collingwood D, Boeck ME, Fox LA, Alvino GM, Fangman WL, et al. Genomic mapping of single-stranded DNA in hydroxyureachallenged yeasts identifies origins of replication. Nat Cell Biol. 2006:8:148-55.

19. Nieduszynski CA, Knox Y, Donaldson AD. Genome-wide identification of replication origins in yeast by comparative genomics. Genes Dev. 2006;20:1874-9.

20. Xu J, Yanagisawa Y, Tsankov AM, Hart C, Aoki K, Kommajosyula N, et al. Genome-wide identification and characterization of replication origins by deep sequencing. Genome Biol. 2012;13:R27.

21. Muller CA, Nieduszynski CA. Conservation of replication timing reveals global and local regulation of replication origin activity. Genome Res. 2012;22:1953-62.

22. Dujon B. Yeast evolutionary genomics. Nat Rev Genet. 2010;11:512-24.

23. Bodey GP, Mardani M, Hanna HA, Boktour M, Abbas J, Girgawy E, et al. The epidemiology of Candida glabrata and Candida albicans fungemia in immunocompromised patients with cancer. Am J Med. 2002;112:380-5.

24. Cormack BP, Ghori N, Falkow S. An adhesin of the yeast pathogen Candida glabrata mediating adherence to human epithelial cells. Science. 1999;285:578-82.

25. Gabaldon T, Martin T, Marcet-Houben M, Durrens P, Bolotin-Fukuhara M, Lespinet $\mathrm{O}$, et al. Comparative genomics of emerging pathogens in the Candida glabrata clade. BMC Genomics. 2013;14:623.

26. Thierry A, Bouchier C, Dujon B, Richard G-F. Megasatellites: a peculiar class of giant minisatellites in genes involved in cell adhesion and pathogenicity in Candida glabrata. Nucl Acids Res. 2008;36:5970-82.

27. Thierry A, Dujon B, Richard GF. Megasatellites: a new class of large tandem repeats discovered in the pathogenic yeast Candida glabrata. Cell Mol Life Sci. 2009;67:671-6.

28. Rolland T, Dujon B, Richard GF. Dynamic evolution of megasatellites in yeasts. Nucleic Acids Res. 2010;38:4731-9.

29. Tekaia F, Dujon B, Richard GF. Detection and characterization of megasatellites in orthologous and nonorthologous genes of 21 fungal genomes. Eukaryot Cell. 2013;12:794-803.

30. Muller H, Thierry A, Coppée J-Y, Gouyette C, Hennequin C, Sismeiro O, et al. Genomic polymorphism in the population of Candida glabrata: gene copy-number variation and chromosomal translocations. Fungal Genet Biol. 2009. doi:10.1016/j.fgb.2008.11.006.

31. Polakova S, Blume C, Zarate JA, Mentel M, Jorck-Ramberg D, Stenderup J, et al. Formation of new chromosomes as a virulence mechanism in yeast Candida glabrata. Proc Natl Acad Sci U S A. 2009;106:2688-93.

32. Muller H, Hennequin C, Galland J, Dujon B, Fairhead C. The asexual yeast Candida glabrata maintains distinct a and alpha haploid mating types. Eukaryot Cell. 2008;7:848-58.

33. Marbouty M, Ermont C, Dujon B, Richard GF, Koszul R. Purification of G1 daughter cells from different Saccharomycetes species through an optimized centrifugal elutriation procedure. Yeast. 2014;31:159-66. 
34. Kaykov A, Nurse P. The spatial and temporal organization of origin firing during the S-phase of fission yeast. Genome Res. 2015;25:391-401.

35. Leh Louis V, Despons L, Friedrich A, Martin T, Durrens P, Casarégola S, et al Pichia sorbitophila, an interspecies yeast hybrid, reveals early steps of genome resolution after polyploidization. G3 (Bethesda). 2012;2:299-311.

36. Morales L, Dujon B. Evolutionary role of interspecies hybridization and genetic exchanges in yeasts. Microbiol Mol Biol Rev. 2012;76:721-39.

37. Gordon JL, Byrne KP, Wolfe KH. Mechanisms of chromosome number evolution in yeast. PLoS Genet. 2011;7, e1002190.

38. Chang F, Theis JF, Miller J, Nieduszynski CA, Newlon CS, Weinreich M. Analysis of chromosome III replicators reveals an unusual structure for the ARS318 silencer origin and a conserved WTW sequence within the origin recognition complex binding site. Mol Cell Biol. 2008;28:5071-81.

39. Ganley AR, Kobayashi T. Highly efficient concerted evolution in the ribosomal DNA repeats: total rDNA repeat variation revealed by whole-genome shotgun sequence data. Genome Res. 2007;17:184-91.

40. James SA, O'Kelly MJ, Carter DM, Davey RP, van Oudenaarden A, Roberts IN. Repetitive sequence variation and dynamics in the ribosomal DNA array of Saccharomyces cerevisiae as revealed by whole-genome resequencing. Genome Res. 2009;19:626-35.

41. Proux-Wera E, Byrne KP, Wolfe KH. Evolutionary mobility of the ribosomal DNA array in yeasts. Genome Biol Evol. 2013;5:525-31.

42. Wyrick JJ, Aparicio JG, Chen T, Barnett JD, Jennings EG, Young RA, et al. Genome-wide distribution of ORC and MCM proteins in S. cerevisiae: high-resolution mapping of replication origins. Science. 2001;294:2357-60.

43. Witten DM, Noble WS. On the assessment of statistical significance of threedimensional colocalization of sets of genomic elements. Nucleic Acids Res. 2012:40:3849-55.

44. Duan Z, Andronescu M, Schutz K, Mcllwain S, Kim YJ, Lee C, et al. A threedimensional model of the yeast genome. Nature. 2010;465:363-7.

45. Cournac A, Marie-Nelly H, Marbouty M, Koszul R, Mozziconacci J. Normalization of a chromosomal contact map. BMC Genomics. 2012;13:436.

46. Zordan RE, Ren Y, Pan SJ, Rotondo G, De Las Penas A, lluore J, et al. Expression plasmids for use in Candida glabrata. G3 (Bethesda). 2013;3:1675-86.

47. Richard G-F, Kerrest A, Lafontaine I, Dujon B. Comparative genomics of hemiascomycete yeasts: genes involved in DNA replication, repair, and recombination. Mol Biol Evol. 2005;22:1011-23.

48. Richard GF, Kerrest A, Dujon B. Comparative genomics and molecular dynamics of DNA repeats in eukaryotes. Microbiol Mol Biol Rev. 2008:72:686-727.

49. Sutherland GR, Baker E, Richards RI. Fragile sites still breaking. Trends Genet. 1998:14:501-6.

50. Letessier A, Millot GA, Koundrioukoff S, Lachagès A-M, Vogt N, Hansen RS, et al. Cell-type-specific replication initiation programs set fragility of the FRA3B fragile site. Nature. 2011;470:120-3.

51. Yudkin D, Hayward BE, Aladjem MI, Kumari D, Usdin K. Chromosome fragility and the abnormal replication of the FMR1 locus in fragile $X$ syndrome. Hum Mol Genet. 2014;23:2940-52.

52. Fraser HB. Cell-cycle regulated transcription associates with DNA replication timing in yeast and human. Genome Biol. 2013:14:R111.

53. Omberg L, Meyerson JR, Kobayashi K, Drury LS, Diffley JF, Alter O. Global effects of DNA replication and DNA replication origin activity on eukaryotic gene expression. Mol Syst Biol. 2009;5:312.

54. Tsankov AM, Thompson DA, Socha A, Regev A, Rando OJ. The role of nucleosome positioning in the evolution of gene regulation. PLOS Biol. 2010;8, e1000414.

55. Haase SB, Reed SI. Improved flow cytometric analysis of the budding yeast cell cycle. Cell Cycle. 2002;1:132-6.

56. FASTX Toolkit package. http://hannonlab.cshl.edu/fastx_toolkit/

57. Dujon B, Sherman D, Fischer G, Durrens P, Casaregola S, Lafontaine I, et al. Genome evolution in yeasts. Nature. 2004;430:35-44.

58. Génolevures: Complete Genomes. http://www.genolevures.org/download. html\#cagl

59. Li H, Durbin R. Fast and accurate short read alignment with Burrows-Wheeler transform. Bioinformatics. 2009:25:1754-60.

60. Li H, Handsaker B, Wysoker A, Fennell T, Ruan J, Homer N, et al. The Sequence Alignment/Map format and SAMtools. Bioinformatics. 2009;25:2078-9.

61. Millot G. Comprendre et réaliser les tests statistiques à l'aide de R. 2nd ed. Brussels: De Boeck; 2011.
62. Press WH, Teukolsky SA, Vetterling WT, Flannery BP. Numerical recipes. The art of scientific computing. 3rd ed. New York: Cambridge University Press; 2007.

63. Cleveland WS. LOWESS - a program for smoothing scatterplots by robust locally weighted regression. Am Stat. 1981;35:54-4.

64. Brent R. Algorithms for minimization without derivatives. New Jersey: Prentice-Hall; 1973.

65. Inoue $\mathrm{H}$, Nojima $\mathrm{H}$, Okayama $\mathrm{H}$. High efficiency transformation of Escherichia coli with plasmids. Gene. 1990;96:23-8.

66. Sambrook J, Russell DW. The inoue method for preparation and transformation of competent E. coli: "ultra-competent" cells. CSH Protoc. 2006, 2006: doi:10.1101/pdb.prot3944.

67. Annaluru N, Muller H, Mitchell LA, Ramalingam S, Stracquadanio G, Richardson SM, et al. Total synthesis of a functional designer eukaryotic chromosome. Science. 2014;344:55-8.

68. Ng P, Keich U. GIMSAN: a Gibbs motif finder with significance analysis. Bioinformatics. 2008;24:2256-7.

69. Bailey TL, Gribskov M. Combining evidence using p-values: application to sequence homology searches. Bioinformatics. 1998;14:48-54.

70. Engel SR, Dietrich FS, Fisk DG, Binkley G, Balakrishnan R, Costanzo MC, et al, The reference genome sequence of Saccharomyces cerevisiae: then and now. G3 (Bethesda). 2014;4:389-98.

71. Génolevures: Genomic Exploration of the Hemiascomycete Yeasts. http://www.genolevures.org/

72. Marie-Nelly H, Marbouty M, Cournac A, Liti G, Fischer G, Zimmer C, et al. Filling annotation gaps in yeast genomes using genome-wide contact maps. Bioinformatics. 2014;30:2105-13.

73. Langmead B, Salzberg SL. Fast gapped-read alignment with Bowtie 2 . Nat Methods. 2012:9:357-9.

74. Imakaev M, Fudenberg G, McCord RP, Naumova N, Goloborodko A, Lajoie $\mathrm{BR}$, et al. Iterative correction of $\mathrm{Hi}-\mathrm{C}$ data reveals hallmarks of chromosome organization. Nat Methods. 2012;9:999-1003.

75. GitHub repository. https://github.com/sdeclere/cagl_rpl.

76. Muller H, Hennequin C, Dujon B, Fairhead C. Ascomycetes: the Candida MAT locus: comparing MAT in the genomes of hemiascomycetous yeasts. In: Heitman J, editor. Sex in fungi: molecular determination and evolutionary implications. Washington, DC: ASM Press; 2007. p. 247-63.

77. Marbouty M, Cournac A, Flot JF, Marie-Nelly H, Mozziconacci J, Koszul R. Metagenomic chromosome conformation capture (meta3C) unveils the diversity of chromosome organization in microorganisms. Elife. 2014;3, e03318.

\section{Submit your next manuscript to BioMed Central and take full advantage of:}

- Convenient online submission

- Thorough peer review

- No space constraints or color figure charges

- Immediate publication on acceptance

- Inclusion in PubMed, CAS, Scopus and Google Scholar

- Research which is freely available for redistribution 